\title{
Title: Killing of Gram-negative and Gram-positive bacteria by a bifunctional cell wall-targeting T6SS effector
}

\section{Authors:}

Nguyen-Hung Le ${ }^{1}$, Victor Pinedo ${ }^{2}, J^{\prime}$ venal Lopez ${ }^{1}$, Felipe Cava ${ }^{2}$, Mario Feldman ${ }^{*}$

\section{Affiliations:}

${ }^{1}$ Department of Molecular Microbiology, Washington University in St. Louis, School of Medicine, St Louis, MO, 63110, USA.

${ }^{2}$ Laboratory for Molecular Infection Medicine Sweden, Department of Molecular Biology, Umeå Centre for Microbial Research, Umeå University, 90187 Umeå, Sweden.

* Corresponding author: mariofeldman@wustl.edu 


\section{Abstract}

2 The type VI secretion system (T6SS) is a powerful tool deployed by Gram-negative 3 bacteria to antagonize neighboring organisms. Here, we report that Acinetobacter 4 baumannii ATCC 17978 (Ab17978) secretes D-lysine (D-Lys), increasing the 5 extracellular $\mathrm{pH}$ and enhancing the peptidoglycanase activity of the T6SS effector Tse4. 6 This synergistic effect of D-Lys on Tse4 activity enables Ab17978 to outcompete Gram7 negative bacterial competitors, demonstrating that bacteria can modify their

8 microenvironment to increase their fitness during bacterial warfare. Remarkably, this 9 lethal combination also results in T6SS-mediated killing of Gram-positive bacteria. 10 Further characterization revealed that Tse4 is a bifunctional enzyme consisting of both 11 Iytic transglycosylase and endopeptidase activities, thus representing a novel family of 12 modularly organized T6SS PG degrading effectors with an unprecedented impact in 13 antagonistic bacterial interactions.

\section{One sentence summary}

15 By modulating local environmental $\mathrm{pH}$ through D-Lys secretion, Acinetobacter 16 baumannii enhances the activity of a bifunctional cell wall-targeting T6SS effector, 17 increasing its killing activity against Gram-negative and -positive competitors. 


\section{Main text}

19 Bacteria live in dense communities and are often in constant competition with other

20 bacterial species to secure nutrients and space. Bacterial warfare is mediated by the 21 production of diffusible antimicrobial compounds and by sophisticated molecular 22 weapons, such as the type VI secretion system (T6SS). The T6SS is a dynamic 23 nanomachine that delivers toxic effector proteins from an attacking cell (predator) to 24 nearby competitors (prey) in a contact-dependent manner. Although bacteria possess a 25 diverse arsenal of toxic effector proteins to kill Gram-negative bacteria, current evidence 26 suggests that Gram-positive bacteria are immune to T6SS attacks. Immunity to T6SS27 dependent killing between Gram-negative kin cells is accomplished by the expression of 28 immunity proteins, which specifically bind and inactivate their cognate effector. Broad29 spectrum mechanisms of protection against non-kin T6SS attacks in Gram-negative 30 bacteria have only recently been uncovered $(1,2)$.

31 Due to its essentiality, the bacterial cell wall peptidoglycan (PG), also known as murein, 32 is targeted by various T6SS effectors. PG is composed of glycan chains of 33 alternating $\mathrm{N}$-acetylglucosamine (GlcNAc) and $\mathrm{N}$-acetylmuramic acid (MurNAc) that are 34 crosslinked through MurNAc-attached peptides. PG-degrading (PGase) effectors induce 35 bacterial cell lysis, which facilitates the complete clearance of bacterial competitors by 36 preventing dead cells from shielding susceptible prey from T6SS attacks, a 37 phenomenon known as the "corpse barrier" effect(3). The PGase effectors 38 characterized to date contain one lytic enzymatic activity and can be categorized as 39 muramidases, which cleave the $\beta$ - $(1,4)$-glycosidic bond $(4,5)$, N-acetylmuramyl-L40 alanine amidases, which unlink the peptides chains from MurNAc (6) and LD- (4, 7), or 41 DD-endopeptidases( 7 ), which cleave PG crosslinks.

42 T6SS-dependent bacterial warfare generates an arms race in which both predator and 43 prey evolve novel tools to prevail. We recently showed that some Acinetobacter 44 baumannii strains, such as Ab17978, use the periplasmic racemase Rack to produce 45 the non-canonical D-amino acid (NCDAA) D-lysine (D-Lys). Incorporation of D-Lys into 46 the PG of Ab17978 underlies a defensive strategy against the PGase activity of T6SS 47 effectors from competing bacteria(2). Beyond being incorporated into the PG, most of 
48 the D-Lys that is produced is secreted into the extracellular milieu, where it accumulates

49 to millimolar concentrations(2). It has been recently suggested that NCDAAs carry out

50 diverse biological roles in bacterial ecosystems(8). Therefore, we decided to investigate

51 the role of extracellular D-Lys in bacterial warfare.

52 Previous work has shown that T6SS-dependent toxicity is impacted by the extracellular 53 environment(9). We hypothesized that D-Lys secretion could potentiate T6SS-

54 dependent bacterial killing by Ab17978. To test this hypothesis, we compared the T6SS

55 killing activity of wild-type (WT) Ab17978 with that of its racK deletion ( $\triangle$ racK) derivative.

56 We found that the T6SS of WT Ab17978 is 100 fold more lethal than the $\Delta$ racK strain

57 against two different prey, Escherichia coli MG1655 and Acinetobacter nosocomialis M2

58 with an inactive T6SS (M2 $\Delta$ tssB) (Fig. 1A). As expected, Ab17978 $\Delta$ tss $M$, which

59 possesses an inactive T6SS, did not display bacterial killing (Fig. 1A). WT and $\Delta$ racK

60 secreted equal amounts of the T6SS protein Hcp, indicating that deletion of racK does

61 not affect T6SS dynamics (Fig. S1). We also confirmed that Ab17978 $\Delta$ tssM secretes

62 similar amounts of D-Lys compared with the WT strain, suggesting that T6SS

63 functionality and D-Lys secretion are independent processes (Fig. S2). Remarkably,

64 heterologous rack expression in the clinical isolate $A$. baumannii strain UPAB1, which

65 does not encode racK, resulted in a 100-fold increase in T6SS lethality compared with

66 WT (Fig. 1B). Together, these data demonstrate that D-Lys secretion enhances the

67 T6SS-mediated killing of $A$. baumannii.

68 Ab17978 encodes four T6SS effectors: Tse1 is a predicted lipase; Tse2 is a predicted 69 nuclease; Tse3 is an effector of unknown function; and Tse4 (ACX60_00605) is 70 predicted to contain PGase activity (10). To gain insight into the effectors involved in D-

71 Lys-mediated enhancement of T6SS lethality, we tested the killing efficiency of mutants

72 unable to secrete one or more effectors. We found that a mutant strain unable to 73 secrete effectors Tse1, Tse2 and Tse3 $(\Delta 123)$ retained most of its killing ability against

$74 \mathrm{M} 2 \Delta t s s B$, indicating that Tse4 plays a major role in bacterial killing (Fig. 2). A $\Delta$ rack 75 derivative of the $\Delta 123$ strain $(\Delta 123 \Delta$ racK), lost most of its killing capacity, indicating that 76 RacK enhances Tse4 toxicity. Immunity proteins are generally encoded adjacently to 77 their cognate effector. Indeed, overexpression of tsi4 (ACX60_00610) in M2 $\Delta$ tssB prey 
78 prevented killing by Ab17978 $\Delta 123$, indicating that Tsi4 is the immunity protein of Tse4

79 (Fig. 2). Furthermore, the enhanced killing provided by RacK disappeared when

$80 \mathrm{M} 2 \Delta t s s B$ expressed tsi4 (Fig. 2). Together, our results strongly suggest that the

81 synergistic effect of RacK on T6SS lethality is linked to the cell wall-targeting effector

82 Tse4.

83 Next, we performed interbacterial competition assays between the Ab17978 $\Delta$ racK 84 predator and $\mathrm{M} 2 \Delta t s s B$ prey in media supplemented with increasing amounts of D-Lys.

85 Consistent with our previous result, we found that T6SS-dependent prey killing was 86 augmented with increasing concentrations of D-Lys. The effect was noticeable at $5 \mathrm{mM}$ 87 D-Lys and led to WT killing levels at $20 \mathrm{mM}$ D-Lys (Fig. S3A). Importantly, growth of 88 Ab17978 predator strains and M2 prey strains was not affected by the presence of 20 $89 \mathrm{mM}$ D-Lys in the media (Fig. S5), thus excluding the possible toxicity of D-Lys alone. 90 Extracellular NCDAAs, such as D-Met, can be incorporated into the PG of non91 producing species(11). To test whether the enhanced T6SS lethality observed in the 92 presence of D-Lys can be emulated by other NCDAAs, we performed bacterial killing 93 assays in the presence of D-Met, D-Ala, or D-Arg. While D-Met and D-Ala did not 94 potentiate T6SS lethality (Fig. S3C, D), D-Arg mimicked the effect of D-Lys (Fig. S3B). 95 Unexpectedly, we found that L-Lys and L-Arg also synergized with the T6SS (Fig. S3E).

96 These results indicate that basic amino acids, in either their L- or D- form, enhance the 97 lethality of the T6SS. Thus, we then hypothesized that the RacK-dependent 98 enhancement of interbacterial killing was due to a D-Lys-mediated increase in the $\mathrm{pH}$ at 99 the interface between WT Ab17978 and its prey. Supporting this concept, when killing 100 assays were performed in a buffered $\mathrm{pH}$ of 6.8 (the $\mathrm{pH}$ of LB media) we found that the 101 presence of RacK or 20mM D-Lys no longer increased the bactericidal effect of 102 Ab17978 (Fig. 3A, Fig. S4). In contrast, bacterial killing by Ab17978 WT and $\Delta$ racK was 103 increased in a buffered $\mathrm{pH}$ of 8.0 (Fig. 3A). We hypothesized that the increased T6SS104 dependent killing at alkaline $\mathrm{pH}$ could be due to enhanced Tse4 activity in this condition. 105 To this end, we tested the in vitro activity of purified Tse4 using a Remazol Brilliant Blue 106 (RBB)-dye release assay and found that the enzymatic activity of Tse4 was optimal at $107 \mathrm{pH} 8$ and above (Fig. 3B). Based on this data, we propose a model in which D-Lys 
108 secretion increases T6SS-mediated killing by creating an alkaline microenvironment in

109 the predator-prey interface, resulting in maximal Tse4 activity.

110 The dramatic RacK-induced killing efficiency of Ab17978 prompted us to test if this

111 bacterium is able to kill Gram-positive bacteria. Remarkably, we found that Ab17978

112 can kill strains of Bacillus subtilis, Listeria monocytogenes, and methicillin-resistant

113 Staphylococcus aureus (MRSA) in a T6SS- and RacK-dependent manner (Fig. 4A).

114 These Gram-positive prey did not inhibit Ab17978 growth during co-incubation (Fig. S6).

115 Notably, we found that the $\Delta 4$ strain, which lacks $t s e 4$, did not kill any of these strains,

116 while the $\Delta 123$ strain exhibited increased killing in all cases. We hypothesize that the

$117 \Delta 123$ strain secretes increased amounts of Tse4 due to the lack of competition for the

118 T6SS machinery, which is consistent with previous work in Pseudomonas

119 aeruginosa(12). Importantly, addition of purified Tse4 to B. subtilis cultures did not

120 induce cell lysis (Fig. 4B), and co-incubation of Ab17978 and B. subtilis in liquid media

121 did not result in bacterial killing (Fig. S7), indicating that T6SS-dependent activity

122 against Gram-positive bacteria is contact-dependent. Our results are the first

123 demonstration of T6SS-dependent killing of Gram-positive bacteria.

124 As for E. coli, PG of B. subtilis and L. monocytogenes use D-Ala-meso diaminopimelate 125 (mDAP) crosslinks (Fig. 5A)(13). In contrast, S. aureus PG uses a penta-Gly bridge 126 connecting the peptide stems (Fig. 5A)(13). Therefore, Tse4 has the remarkable ability 127 to degrade different PG chemotypes. The Conserved Domain Architecture Retrieval 128 Tool (CDART) algorithm predicted that Tse4 has three conserved domains: a LysM 129 domain in the N-terminal region, followed by a lysozyme-like domain and a Zinc-binding 130 peptidase domain. Consistently, the Phyre2 server identified two conserved catalytic 131 domains in Tse4 (Fig. S8). Amino acid residues 242-447 modeled to a lysozyme-like 132 domain structurally similar to lytic transglycosylase gp144 of bacteriophage phiKZ (PDB: $1333 \mathrm{BKH})$, and this domain would therefore be predicted to non-hydrolytically cleave the 134 glycan chains between GlcNAc and MurNAc, thus producing anhydromuropeptides. 135 Amino acid residues 634-798 modeled to two distinct structures. In the first, the 136 peptidase domain was modeled to the DD-endopeptidase ShyA of Vibrio cholerae 137 (PDB:6U2A), which cleaves D-Ala-mDAP bonds(14). In the second, the peptidase 
138 domain was modeled to the lysostaphin LytM of $S$. aureus (PDB: 2B44), an antibacterial 139 enzyme that is capable of cleaving the penta-Gly bridges found in the PG crosslinks of 140 staphylococci(15). Thus, this analysis suggests that Tse4 is likely a bifunctional (lytic 141 transglycosylase and endopeptidase) effector capable of targeting the cell wall of 142 various bacteria.

143 To determine whether Tse4 possesses lytic transglycosylase and endopeptidase 144 activities, we performed in vitro enzymatic assays followed by analysis of the solubilized 145 muropeptides (PG fragments) by Ultra Performance Liquid Chromatography and Mass 146 Spectrometry (UPLC-MS). Muramidase digestion of the PG produces a profile where 147 the main peaks (muropeptides) are the monomeric disaccharide tetrapeptide (M4) and 148 its dimeric (crosslinked) form (D44 and D44N) (Fig. 5B, S9). Subsequent addition of 149 Tse4 converts the dimers to monomers (M4 and M4N), thereby confirming its 150 endopeptidase activity. To test the putative lytic transglycosylase activity of Tse4, we 151 incubated the PG first with the endopeptidase ShyA, which breaks the crosslinks to 152 produce PG chains of diverse length and always ending with anhydro-M4 (M4N). 153 Subsequent addition of Tse4 further processes the chains to shorter ones of 1 and 2 154 disaccharides long (M4N and M4-M4N) (Fig. 5B, S9), thereby confirming the lytic 155 transglycosylase activity. As Tse4 cannot further break M4-M4N, we conclude that the 156 predicted Lyz-like domain has endolytic transglycosylase activity. These results were 157 recapitulated by digesting PG with Tse4 alone (Fig. 5B, S9). The identity of M4-M4N 158 was confirmed both by MS and by the observation that this chain can be cleaved by 159 muramidase into M4 and M4N (Fig. 5B, Table S1). Together, these data indicate that 160 Tse4 is, to our knowledge, the first identified bifunctional T6SS effector.

161 A bioinformatics analysis identified orthologues of Tse4 in various Acinetobacter, 162 Klebsiella, Yersinia and Enterobacter strains (Fig. 5C). Furthermore, we identified a 163 series of T6SS effectors displaying a modular organization, in which the endopeptidase, 164 lytic transglycosylase and PG-binding domain are linked in different arrangements (Fig. 165 5C). For example, a putative T6SS effector of Burkholderia spp. contains the same 166 three domains but in the reverse order. Additional related effectors were identified in $E$. 167 coli and Salmonella enterica. Thus, Tse4 belongs to a novel broadly distributed class of 
168 T6SS effectors that share a similar modular architecture. These findings suggest that 169 Tse4-like effectors are important mediators of antagonistic bacterial interactions. In 170 general, the modular arrangement of these newly discovered T6SS effectors resemble 171 the organization of phage endolysins. Endolysins appear to have acquired multiple 172 activities to enable host diversification and facilitate adaptation to specific growth phase173 or strain-specific cell wall modifications(16). It is tempting to speculate that multicatalytic 174 T6SS effectors evolved to provide a fitness advantage against diverse bacterial 175 competitors. We propose that the remarkably broad target range and high potency of 176 Tse4 is due to its dual catalytic activity, which might act synergistically to cause 177 localized, lethal damage in the prey cell wall and/or to adjust to the variable PG 178 chemistries of diverse prey.

179 Early work in $V$. cholerae and $P$. aeruginosa suggested that the T6SS targets Gram180 negative but not Gram-positive bacteria $(17,18)$. It was proposed that the PG of Gram181 positive bacteria is too thick to allow the T6SS machinery to deliver toxic effectors at 182 effective concentrations. However, recent work demonstrated that the T6SSs of Serratia 183 marcescens and Klebsiella pneumoniae mediate fungal cell death(19, 20), indicating 184 that T6SS machines are able to penetrate and deliver toxic effectors across the fungal 185 cell wall $(>110 \mathrm{~nm})$, which is thicker than the cell wall of Gram-positive bacteria $(<80 \mathrm{~nm})$. 186 In this work, we show that Ab17978 employs its bifunctional T6SS effector Tse4 to kill 187 various Gram-positive bacteria, including B. subtilis, L. monocytogenes, and S. aureus.

188 The outcome of interbacterial interactions is impacted by the environmental conditions 189 in which these interactions occur(9). Here, we show that by secreting D-Lys, Ab17978 190 modifies its microenvironment to potentiate Tse4 activity and increase T6SS-dependent 191 killing of Gram-negative and -positive prey. Our data suggests that the synergistic effect 192 of D-Lys on Tse4 lethality is likely due to alkalization of the predator-prey zone of 193 contact. Consistently, we found that Tse4 has enhanced activity under alkaline 194 conditions. Thus, our work establishes a novel class of bifunctional, modularly 195 organized, T6SS effectors effective against Gram-positive and -negative bacteria, 196 highlights the role of D-amino acids in modulating the microenvironment, and redefines 197 the function of the T6SS to include the warfare between Gram-negative and Gram- 
bioRxiv preprint doi: https://doi org/10.1101/2021.03.04 433973; this version posted March 4, 2021. The copyright holder for this preprint (which was not certified by peer review) is the author/funder, who has granted bioRxiv a license to display the preprint in perpetuity. It is made available under aCC-BY-NC-ND 4.0 International license.

198 positive bacteria. Future work will be necessary to better understand the full scope of 199 T6SS-mediated killing of Gram-negative and -positive bacteria and elucidate the effect 200 of these interactions in shaping the composition of bacterial communities in the context 201 of the human microbiota and polymicrobial infections. 


\section{Acknowledgements}

203 This work was supported by the National Institutes of Health grant 1R01Al125363-01 to 204 M.F.F. Work in the Cava lab was supported by the Swedish Research Council (VR), the 205 Knut and Alice Wallenberg Foundation (KAW), the Laboratory of Molecular Infection 206 Medicine Sweden (MIMS), and the Kempe Foundation. We thank Dr. Ichiro Matsumura 207 (Emory University) for providing B. subtilis SCK6 harboring pBAV1k-T5-gfp plasmid. We 208 thank Dr. Laura Alvarez for providing ShyA. We thank Dr. Clay Jackson-Litteken for 209 critical reading of the manuscript.

\section{Author Contributions}

211 Conceived and designed the experiments: N.H.L., V.P., F.C. and M.F.F. Performed the 212 experiments: N.H.L., V.P. and J.L. Analyzed the data: N.H.L., V.P., F.C. and M.F.F. 213 Wrote the paper: N.H.L., J.L., and M.F.F.

\section{Declaration of Interests}

215 All authors declare no competing interests. 


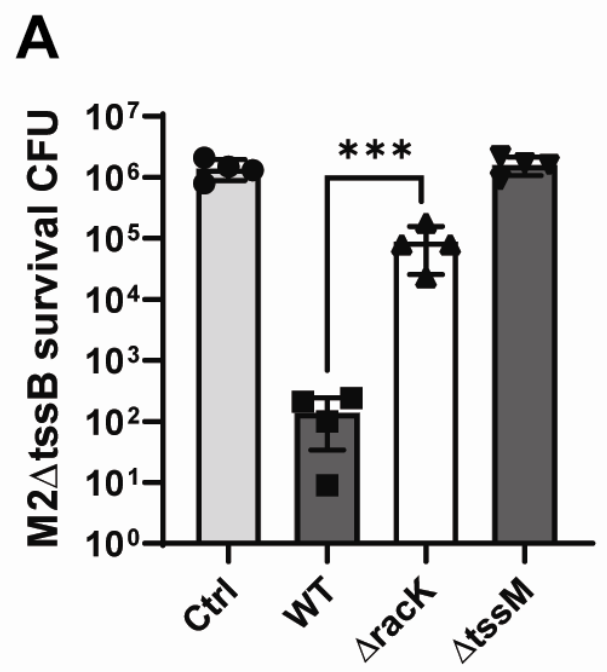

Predator: Ab17978

B

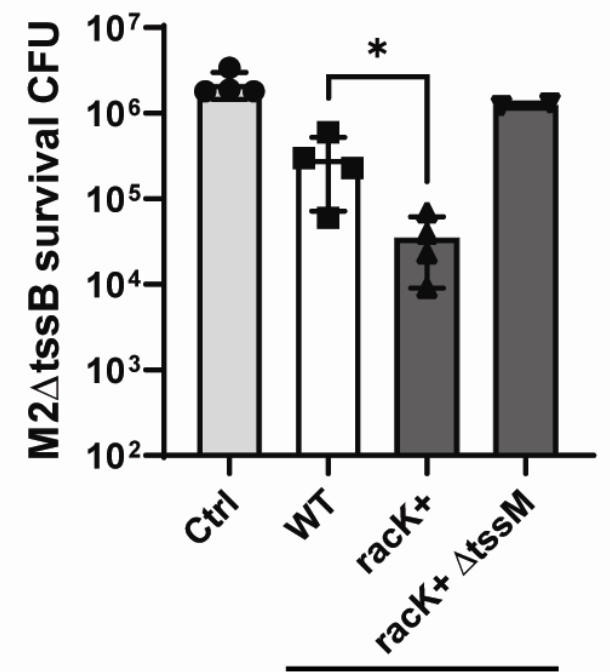

Predator: UPAB1

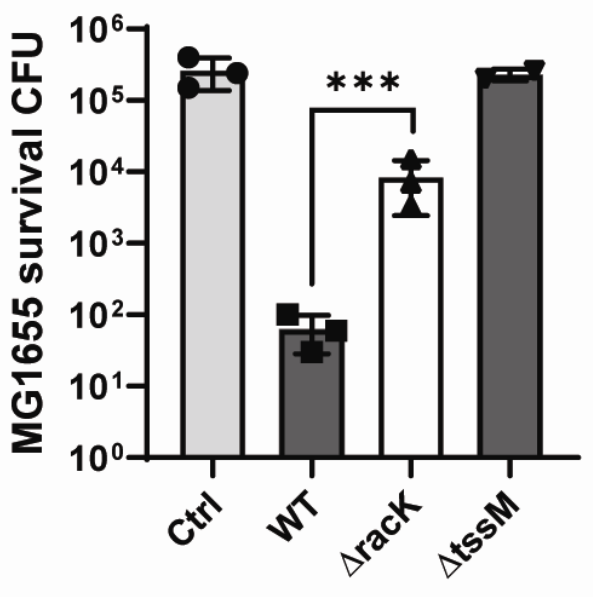

Predator: Ab17978

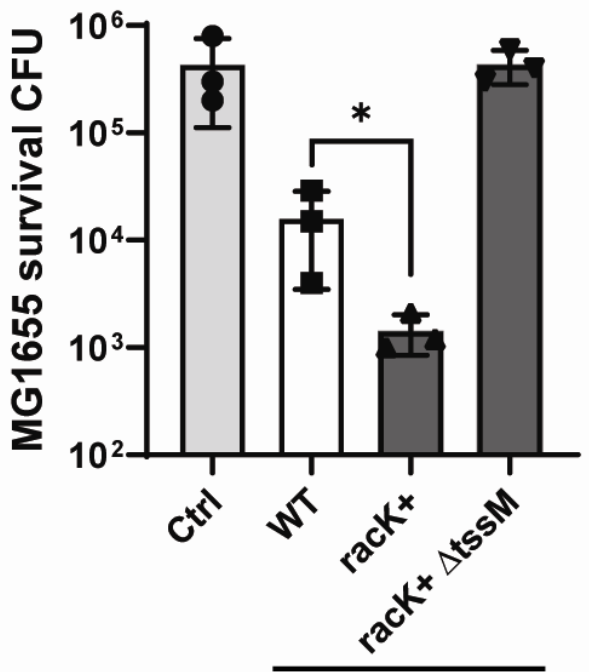

Predator: UPAB1

216 Fig. 1: RacK enhances T6SS-dependent killing by A. baumannii. A. Competition 217 assay using Ab17978 WT, $\Delta$ racK, or $\Delta t s s M$ as predators and $A$. nocosomialis M2 $\Delta$ tss $B$ 218 or E. coli MG1655 as prey. B. Competition assay using A. baumannii UPAB1 WT, 219 UPAB1 heterologously expressing racK $(\mathrm{racK}+)$ or racK $+\Delta t s s M$ as predators and $A$. 220 nocosomialis M2AtssB or E. coli MG1655 as prey. Bar graphs represent means of prey 221 survival CFU after $4 \mathrm{~h}$ of co-incubation \pm SD of at least three biological replicates. 222 Statistical analyses were performed using the unpaired Student's $t$ test, ${ }^{*} p<0.05$ and $223^{* * *} p<0.001$. 


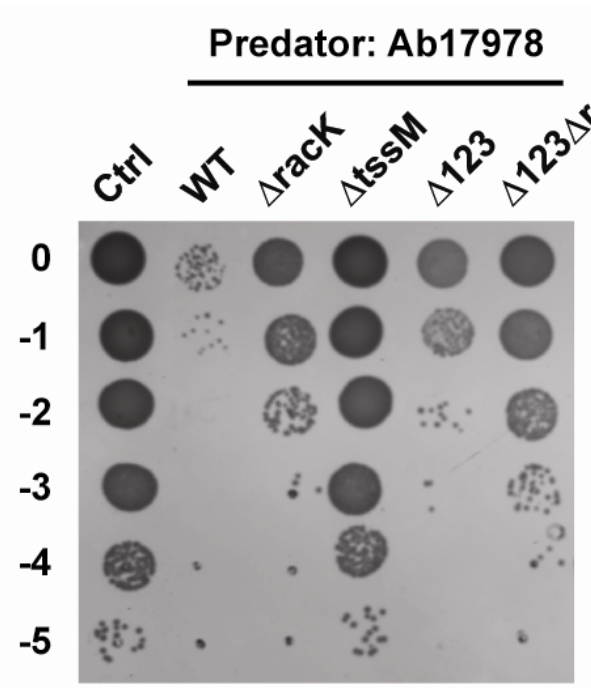

M2 $\Delta$ tss B survival CFU

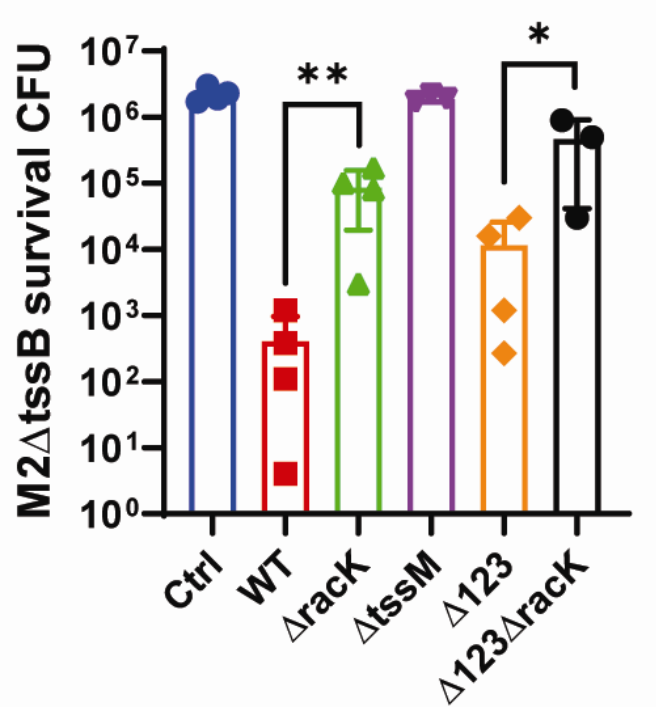

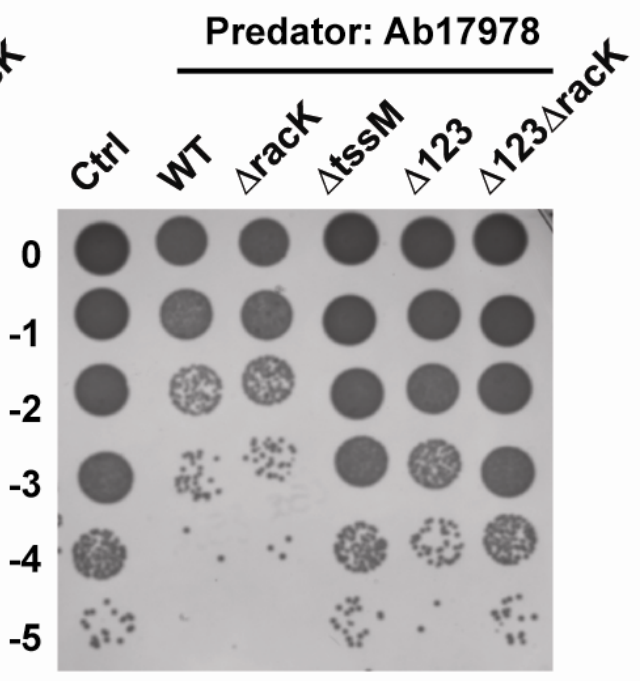

M2 $\Delta$ tssB::tsi4 survival CFU

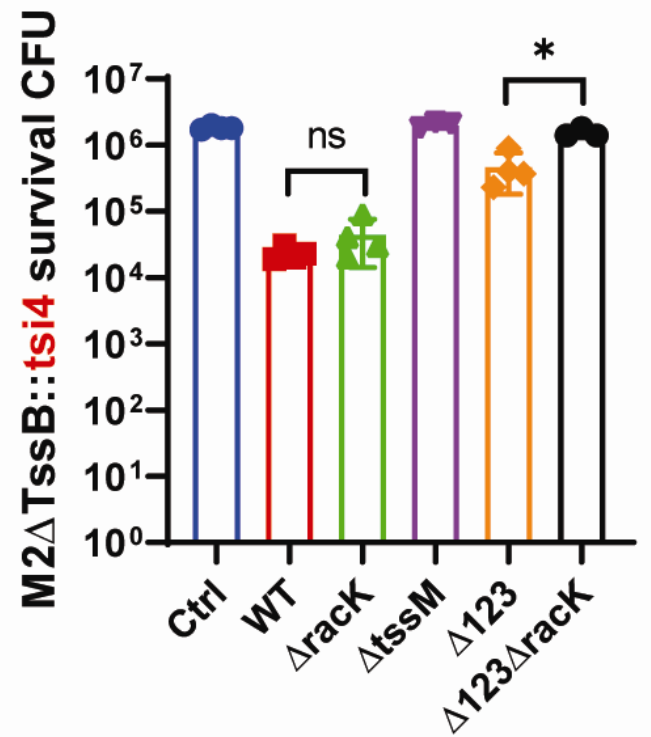

224 Fig. 2: Synergistic effect of RacK on bacterial killing is linked to PG hydrolase 225 effector Tse4. Competition assay using Ab17978 WT, $\Delta$ racK, $\Delta t s s M, \Delta 123, \Delta 123 \Delta$ racK 226 as predators and M2AtssB either harboring an empty vector (left panel) or 227 overexpressing Tsi4, the immunity protein of Tse4 (right panel) as prey. Bar graphs 228 represent means of prey survival CFU after $4 \mathrm{~h}$ of co-incubation \pm SD of at least three 229 biological replicates. Statistical analyses were performed using the unpaired Student's $t$ 230 test, ${ }^{*} p<0.05,{ }^{* *} p<0.01$ and $n s$ not significant. 


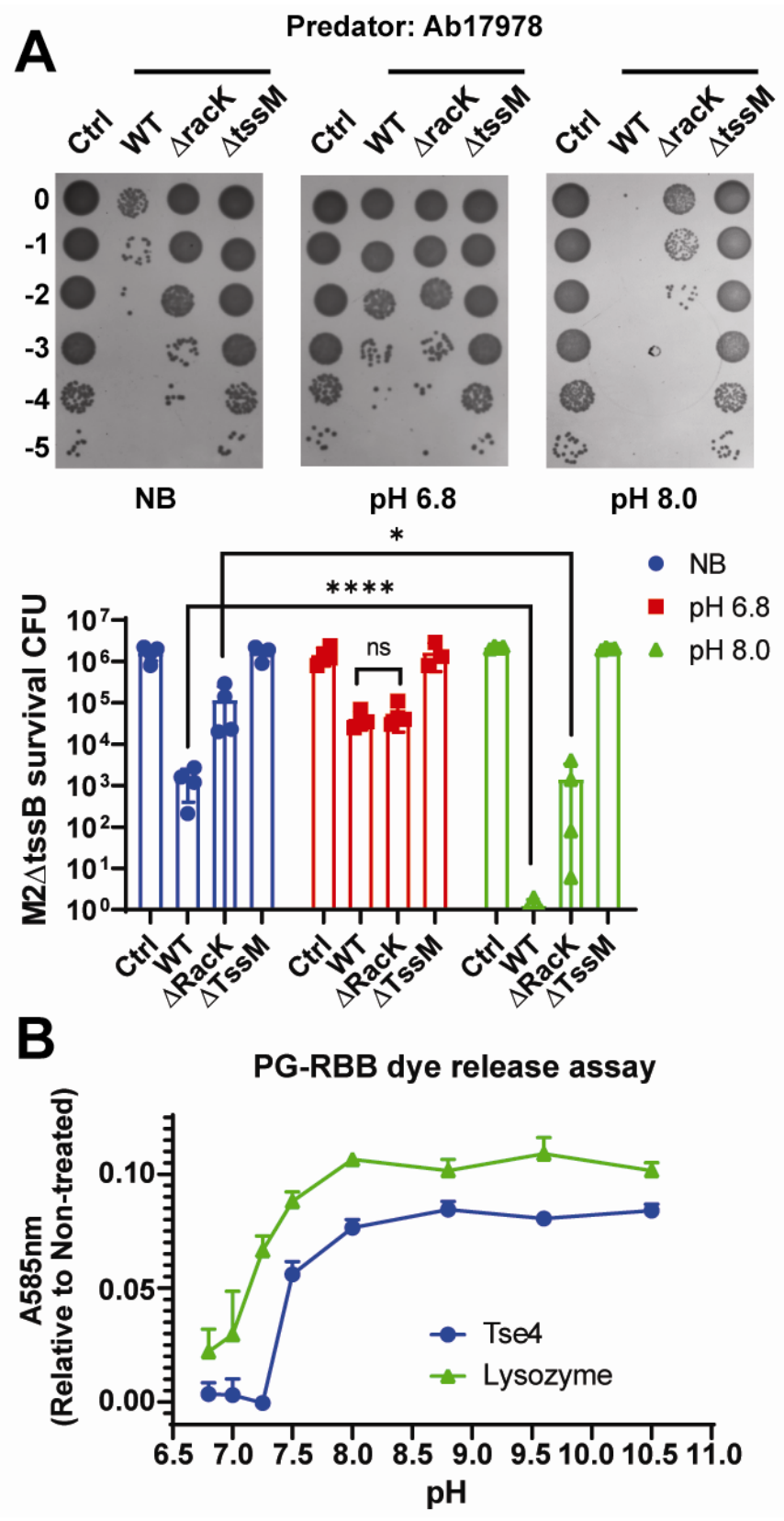

231 Fig. 3: Alkaline environmental conditions enhance bacterial killing and Tse4 232 activity in vitro. A. Competition assay using Ab17978 WT, $\Delta$ rack, or $\Delta t s s M$ as 233 predators and $\mathrm{M} 2 \Delta t s s B$ as prey in non-buffered (NB) media or media buffered at $\mathrm{pH} 6.8$ 234 or 8.0. Bar graphs represent means of prey survival CFU after $4 \mathrm{~h}$ of co-incubation of 235 four biological replicates. B. Remazol Brilliant Blue (RBB)-labeled sacculi were 236 incubated with $7.5 \mu \mathrm{M}$ lysozyme or purified Tse4. After $\sim 16 \mathrm{~h}$, the undigested PG was 237 pelleted and released dye was quantified by measuring dye absorbance at $585 \mathrm{~nm}$. 238 Values represent mean of 3 independent enzymatic assays \pm SD. 

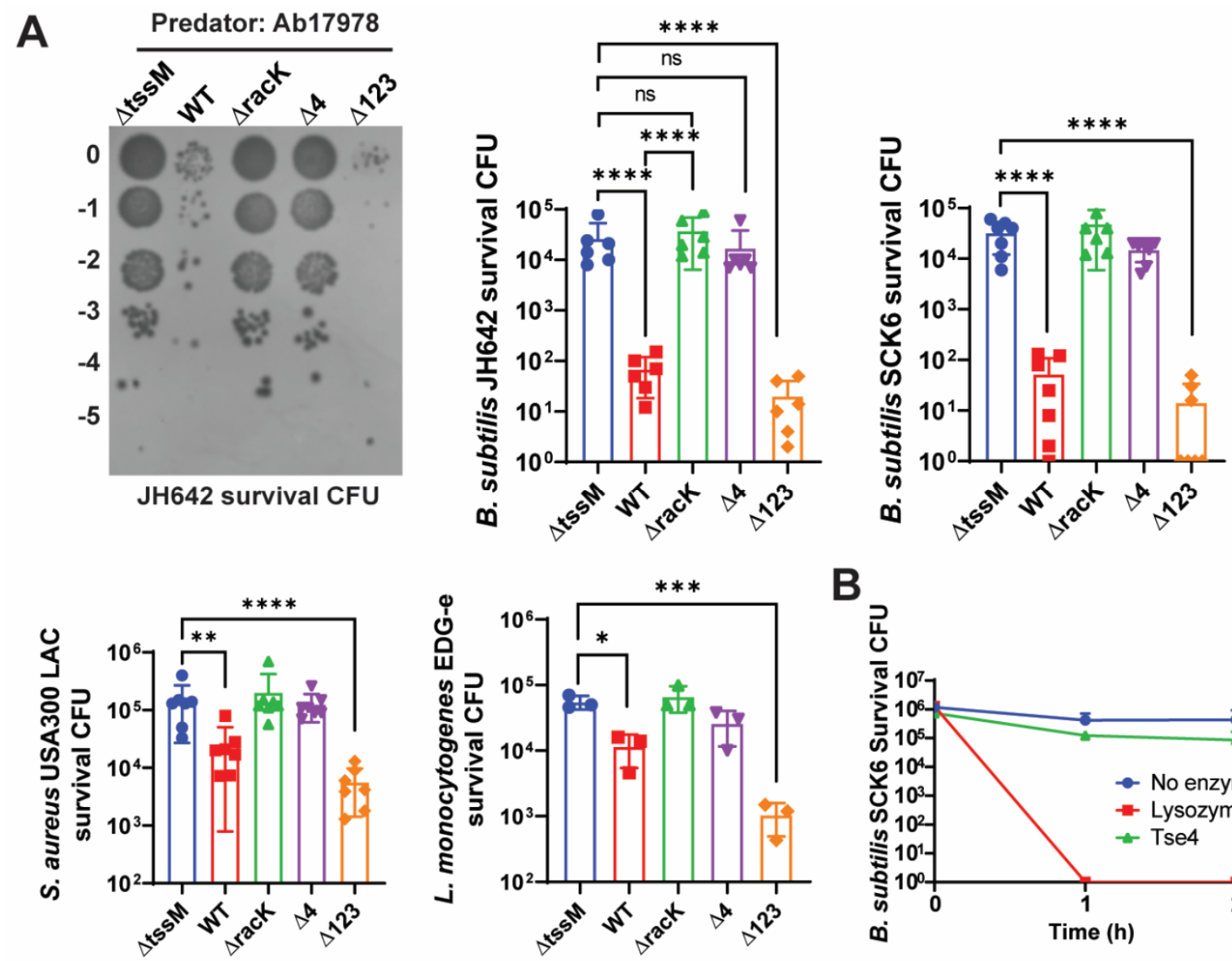

B

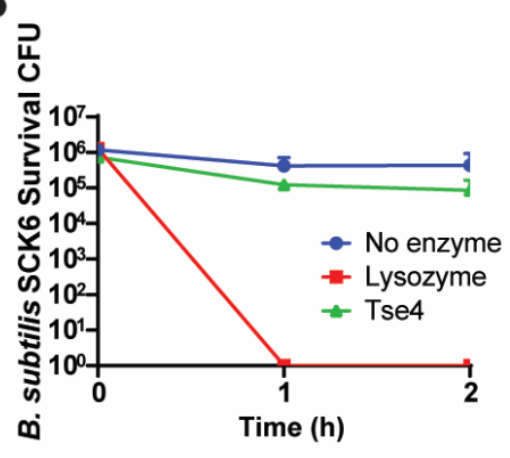

239 Fig. 4: Ab17978 kills various Gram-positive bacteria in a Tse4- and contact240 dependent manner. A. Competition assay using Ab17978 WT, $\Delta t s s M, \Delta r a c K, \Delta 4$ or $241 \Delta 123$ as predators and B. subtilis JH642 or SCK6, S. aureus USA300 LAC, and L. 242 monocytogenes EDG-e as prey. Bar graphs represent means of prey survival CFU after 243 co-incubation \pm SD of at least three biological replicates. Statistical analyses were 244 performed using the unpaired Student's $t$ test, ${ }^{*} p<0.05,{ }^{* *} p<0.01,{ }^{* * *} p<0.001,{ }^{* * *} p<$ 2450.0001 and ns, not significant. B. Late exponential phase growing $B$. subtilis cells 246 (OD600 1) were treated with either $7.5 \mu \mathrm{M}$ of lysozyme or Tse4 for $2 \mathrm{~h}$. Survival CFUs 247 were enumerated at 0,1 and $2 \mathrm{~h}$ of treatment. Values represent means $\pm \mathrm{SD}$ of three 248 biological replicates. 
A

A. nosocomialis $\mathrm{M} 2$, E. coli MG1655, B. subtilis SCK6,

L. monocytogenes EDG-e

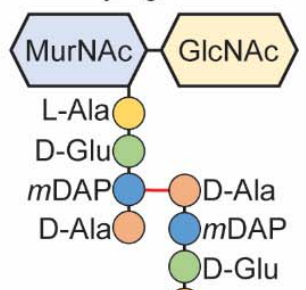

D-Glu

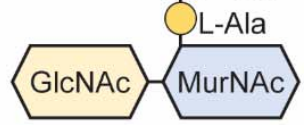

C

Effector organization:
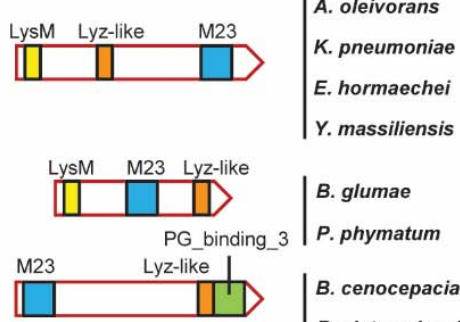

B. cenocepacia B. vietnamiensis \begin{tabular}{l|l|l} 
Lyz-like \\
M23 & S. enterica
\end{tabular} $\square \prod>\left.\right|_{\text {E. coli }}$

vgrG $\square$ transposase
S. aureus USA300 LAC

B

\begin{tabular}{|ccccc|}
\hline$(1)$ & $(2)$ & $(3)$ & $(4)$ & $(5)$ \\
$\mathrm{M} 4$ & $\mathrm{D} 44$ & $\mathrm{D} 44 \mathrm{~N}$ & $\mathrm{M} 4 \mathrm{~N}$ & $\mathrm{M} 4-\mathrm{M} 4 \mathrm{~N}$ \\
$\mathrm{C}$ & & & & \\
\hline & & & & \\
\hline
\end{tabular}

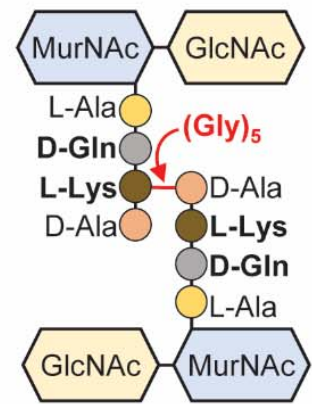

ACX60_00605 DBL02_04225 EON45_RS01265 AC935_RS15280 FTB13_RS16585

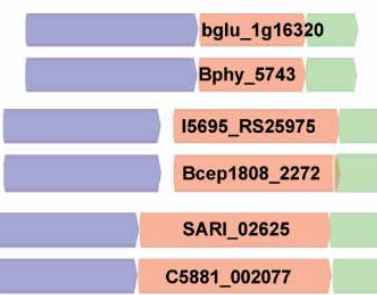

C5881_002077

unknown $\square$ effector

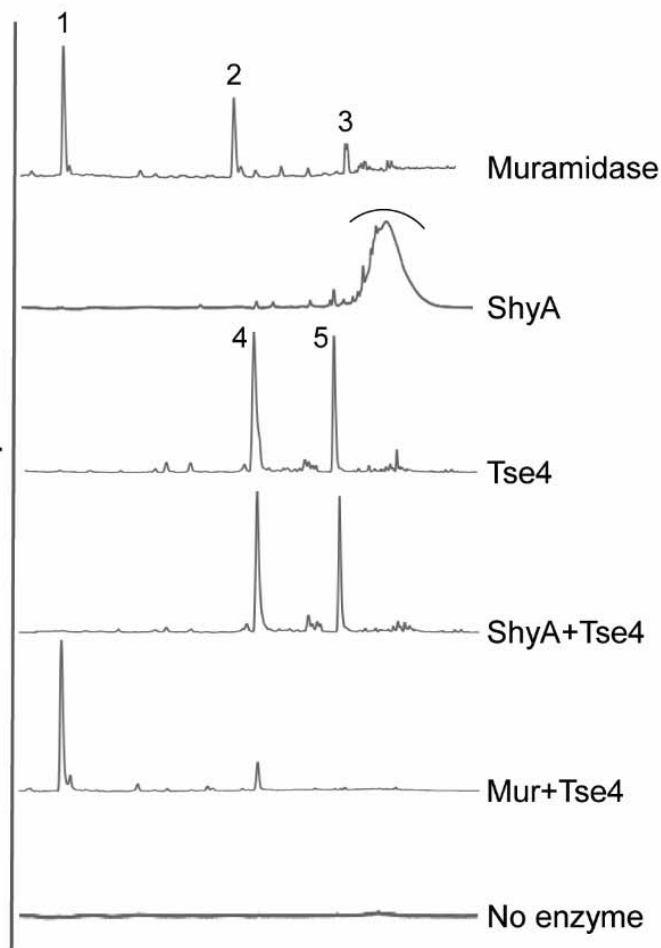

249 Fig. 5: Tse4 is a bifunctional T6SS effector with modular architecture. A. PG 250 composition of bacterial species susceptible to Tse4. B. Chromatograms of 251 muropeptides released following PG treatment with the indicated enzymes. C. Domain 252 architecture of various Tse4-like T6SS effectors. Effector locus tags are indicated. 


\section{Bibliography}

254 1. S. J. Hersch, K. Manera, T. G. Dong, Defending against the Type Six Secretion System: beyond Immunity Genes The bacterial type six secretion system (T6SS) delivers toxic effector proteins into neighboring cells (2020), doi:10.1016/j.celrep.2020.108259.

2. N. H. Le, K. Peters, A. Espaillat, J. R. Sheldon, J. Gray, G. Di Venanzio, J. Lopez, Skaar, F. Cava, W. Vollmer, M. F. Feldman, Peptidoglycan editing provides immunity to Acinetobacter baumannii during bacterial warfare. Sci. Adv. 6, 56145636 (2020).

3. W. P. J. Smith, A. Vettiger, J. Winter, T. Ryser, L. E. Comstock, M. Basler, K. R. Foster, The evolution of the type $\mathrm{VI}$ secretion system as a disintegration weapon. PLoS Biol. 18, e3000720 (2020).

4. A. B. Russell, R. D. Hood, N. K. Bui, M. LeRoux, W. Vollmer, J. D. Mougous, Type VI secretion delivers bacteriolytic effectors to target cells. Nature. 475, 343347 (2011).

5. J. C. Whitney, S. Chou, A. B. Russell, J. Biboy, T. E. Gardiner, M. A. Ferrin, M. Brittnacher, W. Vollmer, J. D. Mougous, Identification, structure, and function of a novel type $\mathrm{VI}$ secretion peptidoglycan glycoside hydrolase effector-immunity pair. J. Biol. Chem. 288, 26616-26624 (2013).

6. J. Ma, M. Sun, Z. Pan, C. Lu, H. Yao, Diverse toxic effectors are harbored by vgrG islands for interbacterial antagonism in type VI secretion system. Biochim.

276 7. A. B. Russell, P. Singh, M. Brittnacher, N. K. Bui, R. D. Hood, M. A. Carl, D. M. Agnello, S. Schwarz, D. R. Goodlett, W. Vollmer, J. D. Mougous, A widespread bacterial type VI secretion effector superfamily identified using a heuristic approach. Cell Host Microbe. 11, 538-549 (2012).

280 8. A. Aliashkevich, L. Alvarez, F. Cava, New insights into the mechanisms and 
biological roles of D-amino acids in complex eco-systems. Front. Microbiol. 9 (2018), p. 683.

283 9. K. D. LaCourse, S. B. Peterson, H. D. Kulasekara, M. C. Radey, J. Kim, J. D.

Mougous, Conditional toxicity and synergy drive diversity among antibacterial effectors. Nat. Microbiol. 3, 440-446 (2018).

10. B. S. Weber, S. W. Hennon, M. S. Wright, N. E. Scott, V. de Berardinis, L. J. Foster, J. A. Ayala, M. D. Adams, M. F. Feldman, Genetic dissection of the type VI secretion system in Acinetobacter and identification of a novel peptidoglycan hydrolase, TagX, required for its biogenesis. MBio. 7 (2016), doi:10.1128/mBio.01253-16.

11. F. Cava, M. A. de Pedro, H. Lam, B. M. Davis, M. K. Waldor, Distinct pathways for modification of the bacterial cell wall by non-canonical D-amino acids. EMBO J. 30, 3442-3453 (2011).

12. J. C. Whitney, C. M. Beck, Y. A. Goo, A. B. Russell, B. N. Harding, J. A. De Leon, 296 D. A. Cunningham, B. Q. Tran, D. A. Low, D. R. Goodlett, C. S. Hayes, J. D. Mougous, Genetically distinct pathways guide effector export through the type VI secretion system. Mol. Microbiol. 92, 529-542 (2014).

300 14. T. Dörr, F. Cava, H. Lam, B. M. Davis, M. K. Waldor, Substrate specificity of an 301 elongation-specific peptidoglycan endopeptidase and its implications for cell wall architecture and growth of Vibrio cholerae. Mol. Microbiol. 89, 949-962 (2013).

15. M. Firczuk, A. Mucha, M. Bochtler, Crystal structures of active LytM. J. Mol. Biol. 354, 578-590 (2005).

305 16. H. Oliveira, L. D. R. Melo, S. B. Santos, F. L. Nobrega, E. C. Ferreira, N. Cerca, J. 306 Azeredo, L. D. Kluskens, Molecular Aspects and Comparative Genomics of Bacteriophage Endolysins. J. Virol. 87, 4558-4570 (2013).

308 17. D. L. Maclntyre, S. T. Miyata, M. Kitaoka, S. Pukatzki, The Vibrio cholerae type VI 
secretion system displays antimicrobial properties. Proc. Natl. Acad. Sci. U. S. A. 107, 19520-19524 (2010).

311 18. S. Chou, N. K. Bui, A. B. Russell, K. W. Lexa, T. E. Gardiner, M. LeRoux, W. Vollmer, J. D. Mougous, Structure of a Peptidoglycan Amidase Effector Targeted to Gram-Negative Bacteria by the Type VI Secretion System. Cell Rep. 1, 656664 (2012).

315 19. K. Trunk, J. Peltier, Y. C. Liu, B. D. Dill, L. Walker, N. A. R. Gow, M. J. R. Stark, J. Quinn, H. Strahl, M. Trost, S. J. Coulthurst, The type VI secretion system deploys antifungal effectors against microbial competitors. Nat. Microbiol. 3, 920-931 (2018).

319 20. D. Storey, A. McNally, M. Åstrand, J. P. G. Santos, I. Rodriguez-Escudero, B. Elmore, L. Palacios, H. Marshall, L. Hobley, M. Molina, V. J. Cid, T. A. Salminen, microbial competition is PhoPQ controlled and reactive oxygen species dependent. PLoS Pathog. 16 (2020), doi:10.1371/journal.ppat.1007969. 


\section{Supplementary Materials for}

\section{Title: Killing of Gram-negative and Gram-positive bacteria by a bifunctional cell wall-targeting T6SS effector}

\section{Authors:}

Nguyen-Hung Le ${ }^{1}$, Victor Pinedo ${ }^{2}$, Juvenal Lopez ${ }^{1}$, Felipe Cava ${ }^{2}$, Mario Feldman ${ }^{1 *}$

\section{Affiliations:}

1 Department of Molecular Microbiology, Washington University School of Medicine, St Louis, MO, 63110, USA.

${ }^{2}$ Laboratory for Molecular Infection Medicine Sweden, Department of Molecular Biology, Umeå Centre for Microbial Research, Umeå University, 90187 Umeå, Sweden.

* Corresponding author: mariofeldman@wustl.edu

This file includes:

Materials and Methods

Figs. $\mathrm{S} 1$ to $\mathrm{S} 9$

Tables S1 to S3 


\section{Materials and Methods}

325 Bacterial strains and growth conditions

$326 \quad$ Bacterial strains used in this study are listed in Supplementary Table S2. Unless otherwise

327 noted, strains were grown in lysogeny broth (LB) liquid medium at $37 \square^{\circ} \mathrm{C}$ with shaking

328 (200 $\square \mathrm{rpm})$. The antibiotics kanamycin (25 or $50 \square \mu \mathrm{g} / \mathrm{ml})$, gentamicin $(20 \mu \mathrm{g} / \mathrm{ml})$,

329 chloramphenicol (15 $\square \mathrm{\mu g} / \mathrm{ml})$ and carbenicillin $(100 \mu \mathrm{g} / \mathrm{ml})$ were added when necessary.

330 Construction of $A$. baumannii mutant strains

331 The primers used in this study are listed in Supplementary Table S3. Ab17978 $\Delta$ tse4, $\Delta$ racK,

$332 \mathrm{UPAB} 1 \Delta t s s M$ and UPAB1racK+ $\Delta t s s M$ deletion mutants were constructed as described

333 previously(1). Briefly, an antibiotic resistance cassette was amplified with primers pair P1 \& P2

334 (Integrated DNA Technologies) with homology to the flanking regions of the targeted gene with

335 additional 3' 20 nucleotides of homology to the FRT site-flanked kanamycin resistance cassette

336 from plasmid pKD4. This PCR product was electroporated into competent Ab17978,

337 Ab17978 123 unmarked mutant, UPAB1 or UPAB1racK+ carrying plasmid pAT04, which

338 expresses the RecAB recombinase. Mutants were selected on $10 \square \mu \mathrm{g} / \mathrm{ml} \mathrm{kanamycin}$, and

339 integration of the resistance marker was confirmed by PCR. To remove the kanamycin

340 resistance cassette, electrocompetent mutants were transformed with plasmid pAT03, which

341 expresses the FLP recombinase.

342 The tsi4 gene was PCR amplified from Ab17978 and was cloned into vector pWH1266-

343 promlac by Gibson Assembly (Hi-Fi DNA Assembly mix, NEB). The pWH1266-promlac-

344 Tsi4_Ab17978 vector was electroporated into $M 2 \Delta t s s B$ and selected on $15 \mu \mathrm{g} / \mathrm{ml}$ tetracycline,

345 creating the $M 2 \Delta$ tssB::tsi4 strain. 


\section{DAO enzymatic assay}

$3473 \mathrm{~mL}$ of bacterial cultures grown for $20 \mathrm{~h}$ were centrifuged at $10,000 \times \mathrm{g}$ and $0.5 \mathrm{~mL}$ of 348 supernatants were collected. The supernatants were deproteinized using Amicon Ultra-0.5 mL

349 Centrifugal Filters with 3,000 MWCO. The flow through samples were then incubated with 0.4 $350 \mathrm{mg} / \mathrm{ml}$ of $\mathrm{D}$-amino acid oxidase DAO (Sigma-Aldrich) for $1 \mathrm{~h}$ at $37^{\circ} \mathrm{C}$. Hydrogen peroxide co-

351 product was then measured using HyPerBlu chemiluminescent detection kit (Lumigen), 352 according to the manufacturer's instructions.

353 Hcp secretion and Western blotting

354 Ab17978 overnight cultures were back-diluted in fresh LB medium to an OD600 of 0.025

355 and grown at $37^{\circ} \mathrm{C}$ with shaking until they reached an OD600 of 0.4 to 0.7 . The cells were then

356 pelleted by centrifugation. The cells were resuspended in Laemmli buffer to a final OD600 of 10.

357 Supernatant proteins were subsequently precipitated with trichloroacetic acid, as previously 358 described(2). Optical density-normalized volumes of whole cells or supernatants were loaded

359 onto $15 \%$ SDS-PAGE gels for separation, transferred to a nitrocellulose membrane, and probed

360 with polyclonal rabbit anti-Hcp $(1: 1,000)(39)$, polyclonal rabbit anti-6xHis $(1: 2,000$; Invitrogen,

361 Waltham, MA) or monoclonal mouse anti-RNA polymerase (1:2,600; BioLegend, San Diego,

$362 \mathrm{CA})$. Western blots were then probed with IRDye-conjugated anti-mouse and anti-rabbit 363 secondary antibodies (both at 1:15,000; LI-COR Biosciences, Lincoln, NE) and visualized with 364 an Odyssey CLx imaging system (LI-COR Biosciences).

365 Detection of $\mathrm{Hcp}$ in solid growth was done by colony blot(3). $10 \mu \mathrm{L}$ of overnight cultures 366 normalized to OD600 of 1.0 were spotted onto $0.4 \mu \mathrm{m}$ nitrocellulose membrane on top of an LB367 agar (3\%) plate. After $4 \mathrm{~h}$ of incubation at $37 \square \mathrm{C}$, the membrane was washed and blotted as 368 described above. Western-blot signals were quantified using LI-COR Image Studio software. 


\section{Protein expression and purification}

The tse 4 gene was PCR amplified from Ab17978 and cloned into vector pET22b+ void of

371 the pelB sequence using Gibson Assembly (Hi-Fi DNA Assembly mix, NEB). The

372 pET22b+_tse4 vector was electroporated into E. coli Rosetta II (Invitrogen) and selected on

$373100 \square \mu \mathrm{g} / \mathrm{ml}$ carbenicillin. The bacteria were grown in auto-inducible media(4) for $48 \mathrm{~h}$ at $20^{\circ} \mathrm{C}$.

374 Cells were harvested by centrifugation, resuspended in resuspension buffer $(20 \mathrm{mM}$ Tris- $\mathrm{HCl}$

$375 \mathrm{pH}$ 7.5, $300 \mathrm{mM} \mathrm{NaCl}, 10 \%$ glycerol, $10 \mu \mathrm{M}$ b-ME, 0.1\% Triton X100, $30 \mathrm{mM}$ imidazole) and

376 lysed by two passages through a cell disruptor at 35,000 psi. (Constant System Itd., Kennesaw,

377 GA). Cell lysates were clarified by centrifugation at $10,000 \mathrm{rpm}$ for $10 \mathrm{~min}$, then passed over a 378 nickel-nitrilotriacetic acid-agarose (Ni-NTA) column (Gold Bio, St. Louis, MO). The column was 379 washed with resuspension buffer containing $60 \mathrm{mM}$ imidazole, and Tse4 was eluted in 380 resuspension buffer containing $150 \mathrm{mM}$ imidazole. Purified Tse4 was buffer exchanged using 381 Sephadex G25 PD10 column and stored in storing buffer (20 mM Tris- $\mathrm{HCl} \mathrm{pH} 7.5,150 \mathrm{mM}$ $382 \mathrm{NaCl}, 10 \%$ glycerol) at $-80^{\circ} \mathrm{C}$.

383 Interbacterial competition assay

384 Interbacterial competition assays in solid media were performed as previously described(5).

385 Briefly, predator and prey overnight cultures were pelleted, washed in fresh LB, and 386 resuspended at an OD600 of 1.0 . The cultures were mixed at a predator:prey ratio of 1:5 387 (Ab17978:M2 $\Delta t s s B$ or UPAB1:M2 $\Delta t s s B), 1: 20$ (Ab17978:MG1655), 1:10 (UPAB1:MG1655), 388 20:1 (Ab17978:JH642, Ab17978:SCK6, Ab17978:USA300 LAC or Ab17978:EDG-e), and $10 \mu l$ 389 drops were spotted onto an LB-agar (3\%) plate. To test the effect of different amino acids (D390 Lys, D-Arg, D-Ala, D-Met, L-Lys or L-Arg (Sigma-Aldrich)) on Ab17978 T6SS-dependent killing 391 capability, LB-agar plates were supplemented with each amino acid to a final concentration of 1 , 3925,10 or $20 \mathrm{mM}$. In the $\mathrm{pH}$-controlled killing assay, LB-agar plates were supplemented with 393 either $100 \mathrm{mM}$ MOPS $\mathrm{pH} 6.8$ or $20 \mathrm{mM}$ HEPES $\mathrm{pH} 8.0$. After $4 \mathrm{~h}$ at $37^{\circ} \mathrm{C}$ (or $5 \mathrm{~h}$ for $\mathrm{L}$. 
394 monocytogenes EDG-e prey), the spots were harvested, resuspended in $0.7 \mathrm{~mL}$ of LB broth, 395 serially diluted and plated on LB-agar plates supplemented with appropriate antibiotics to 396 determine the CFU of surviving prey (Table S2). CFU of surviving Ab17978 predator was also 397 enumerated on chloramphenicol LB-agar plates.

To assess non-contact killing, we performed an interbacterial competition assay in liquid media. Briefly, overnight cultures of predators and prey normalized to OD600 of 1.0 were added 400 at the same ratios used in the competition assay on solid media. Then, the co-cultures were 401 concentrated 10 times by centrifugation and incubated for $4 \mathrm{~h}$ at $37 \square \mathrm{C}$. Finally, $0.7 \mathrm{ml}$ of LB 402 broth was added to $40 \mu \mathrm{L}$ of co-culture, serially diluted and plated on LB-agar plates 403 supplemented with appropriate antibiotics to determine the CFU of surviving prey.

404 Bacterial cell lysis assay

405 B. subtilis SCK6 was grown to an OD600 of $\sim 1.0$. Cells were harvested and washed in 406 reaction buffer $(20 \mathrm{mM}$ Tris $\mathrm{HCl} \mathrm{pH} 8.0,30 \mathrm{mM} \mathrm{NaCl})$. No enzyme (control), lysozyme or Tse4 407 were added to final concentration of $7.5 \mu \mathrm{M}$. B. subtilis was then serially diluted and surviving 408 CFUs were enumerated at time $=0$ (pre-addition of enzymes), $1 \mathrm{~h}$ and $2 \mathrm{~h}$ (post-addition).

409 PG isolation, Remazol Brilliant Blue (RBB)-labeling, Tse4 in vitro reactions and UPLC analysis

$411 \quad$ PG was isolated from a stationary phase Ab17978 culture grown in LB, as previously 412 described(5). Cells were collected by centrifugation for $15 \mathrm{~min}$ at $4^{\circ} \mathrm{C}$ and $7000 \times \mathrm{g}$ and 413 resuspended in $25 \mathrm{mM}$ phosphate buffer $\mathrm{pH} 6(\mathrm{~PB})$ to a final concentration of $0.2 \mathrm{mg} / \mathrm{ml}$. Cell 414 lysis was achieved by adding the cell suspension dropwise to an equal volume of boiling $8 \%$ $415(\mathrm{w} / \mathrm{v})$ SDS under vigorous stirring and boiling the sample for an additional $30 \mathrm{~min}$. After cooling 416 to room temperature, the crude PG was collected by ultracentrifugation for 30 min at $110,000 x$ $417 \mathrm{~g}$ at $25^{\circ} \mathrm{C}$. Pellets were then washed several times with PB to remove SDS, treated with $\alpha-$ 418 amylase $(1 \mathrm{mg} / \mathrm{ml})$ for $1 \mathrm{~h}$ at $37^{\circ} \mathrm{C}$, and treated with Pronase $\mathrm{E}(2 \mathrm{mg} / \mathrm{ml})$ overnight at $60^{\circ} \mathrm{C}$. The 
419 reaction was stopped by boiling the sample with SDS, as described before, and residual SDS 420 was removed by washing several times with PB.

421 RBB labeling of isolated PG was carried out as described previously(6, 7). First, PG was 422 resuspended in $0.02 \mathrm{M} \mathrm{RBB} 0.25 \mathrm{M} \mathrm{NaOH}$ to $12.5 \mathrm{mg} / \mathrm{ml}$ and incubated overnight at $37^{\circ} \mathrm{C}$ with 423 agitation. The solution was then neutralized with $\mathrm{HCl}$ and RBB-labeled PG was pelleted by 424 ultracentrifugation. Excess RBB was removed by repeatedly washing with water until no RBB 425 was detected in the soluble fraction. RBB-labeled PG was then resuspended in $10 \%$ glycerol to $426400 \mathrm{mg}$ of wet pellet per $\mathrm{mL}$ and stored at $-20^{\circ} \mathrm{C} .40 \mathrm{mg} / \mathrm{ml}$ of RBB-dyed sacculi from Ab17978 427 were subjected to overnight reaction at $37^{\circ} \mathrm{C}$ with $7.5 \mu \mathrm{M}$ of $\mathrm{Tse} 4$, lysozyme or no added 428 enzyme (control reaction) in the reaction buffer $(20 \mathrm{mM}$ Tris- $\mathrm{HCl}$ at different $\mathrm{pH}$, ranging from 4296.8 to $10.5,30 \mathrm{mM} \mathrm{NaCl}, 0.1 \mathrm{mM}$ DTT, 0.1\% Triton X100, $5 \mathrm{mM} \mathrm{ZnCl}_{2}$ and $7.5 \mathrm{mM}$ EDTA). The 430 resulting reactions were centrifuged at $14,000 \times \mathrm{g}$ to remove insoluble undigested RBB-dyed 431 PG. The RBB-dye released in the reaction supernatant was quantified by absorbance at 585 $432 \mathrm{~nm}$.

433 Sacculi from Vibrio cholerae stationary phase cells were isolated as previously described(8).

434 In short, PG sacculi were prepared by boiling bacteria in SDS (5\% v/v). SDS was removed by 435 several washes using ultracentrifugation, and the insoluble material was resuspended in MilliQ 436 water. These sacculi were then subjected to overnight reactions with Tse4, ShyA and/or 437 muramidase at $37^{\circ} \mathrm{C}$, using an established protocol(9). For ShyA and Tse4, the in vitro reactions 438 were performed in $100 \mathrm{mM}$ Tris $-\mathrm{HCl} \mathrm{pH} 8$ containing $7.5 \mu \mathrm{M}$ final concentration of enzyme. 439 Muramidase reactions were carried out in MilliQ water or, when combined with Tse4, in 100 mM 440 Tris- $\mathrm{HCl} \mathrm{pH}$ 8. All assays were stopped by incubating the reaction at $100^{\circ} \mathrm{C}$ for 5 minutes. 441 Soluble muropeptides were separated by Ultra Performance Liquid Chromatography (UPLC, 442 Waters) and identified using a MALDI-TOF MS system (Waters). 
bioRxiv preprint doi: https://doi.org/10.1101/2021.03.04.433973; this version posted March 4, 2021. The copyright holder for this preprint (which was not certified by peer review) is the author/funder, who has granted bioRxiv a license to display the preprint in perpetuity. It is made available under aCC-BY-NC-ND 4.0 International license.

\section{Statistical analysis}

444 All statistical analyses were performed using GraphPad Prism 8.0 (GraphPad Software Inc.,

445 La Jolla, CA). For all datasets, Student's unpaired $t$ tests were used. 

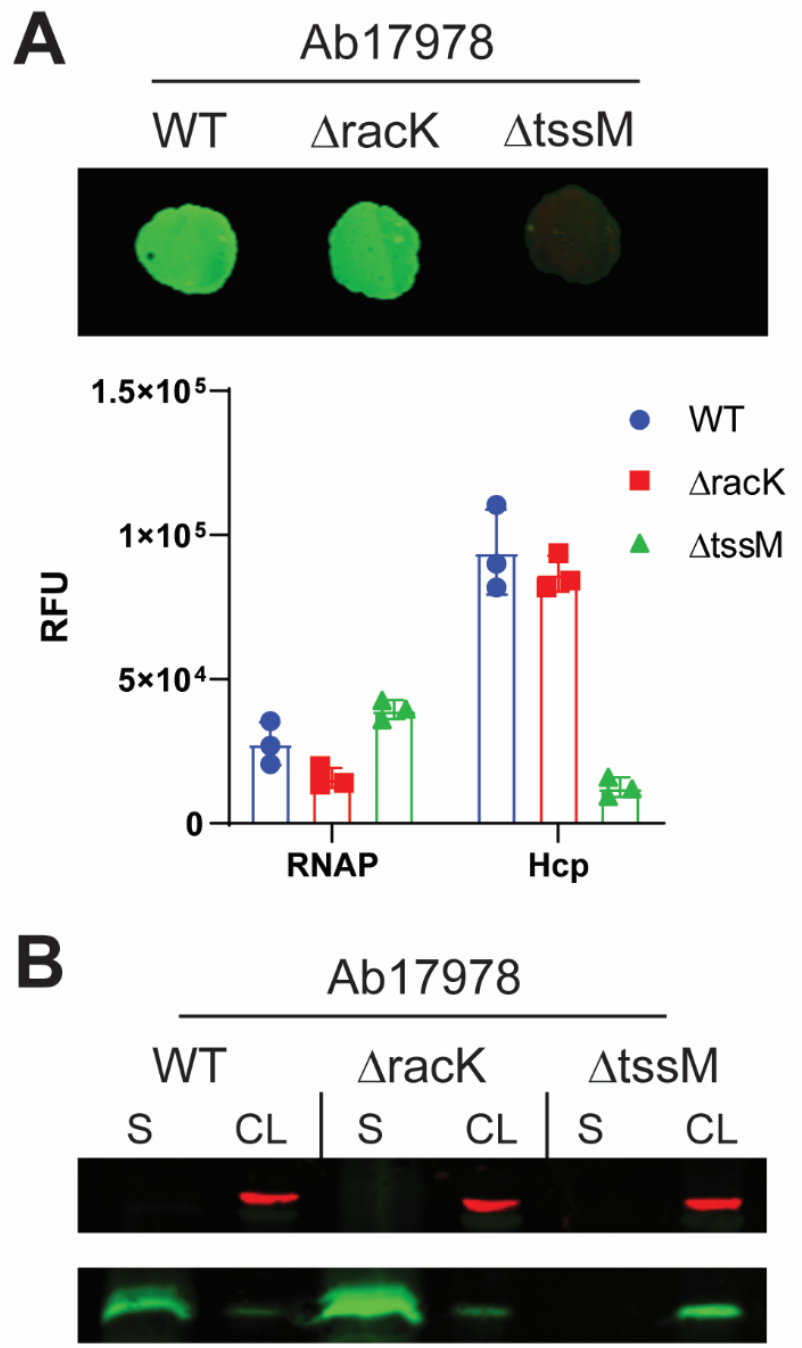

Fig. S1: Deletion of racK does not impair Hcp secretion in Ab17978. A. (top)

447 Representative colony blot of OD-normalized cell cultures of the indicated strains probing for

$448 \mathrm{Hcp}$ and RNA polymerase (RNAP, lysis control). The cells were spotted on LB agar plates and

449 allowed to grow for $4 \mathrm{~h}$ to replicate the killing assay conditions. A high signal of Hcp is indicative

450 of T6SS activity. (bottom) Quantification of three independent colony blot experiments \pm SD. B.

451 Western blot of OD-normalized supernatant $(\mathrm{S})$ and cell lysate $(\mathrm{CL})$ fractions from the indicated

452 Ab17978 strains probing for Hcp expression and secretion (green). RNAP is used as a lysis and 453 loading control (red). 


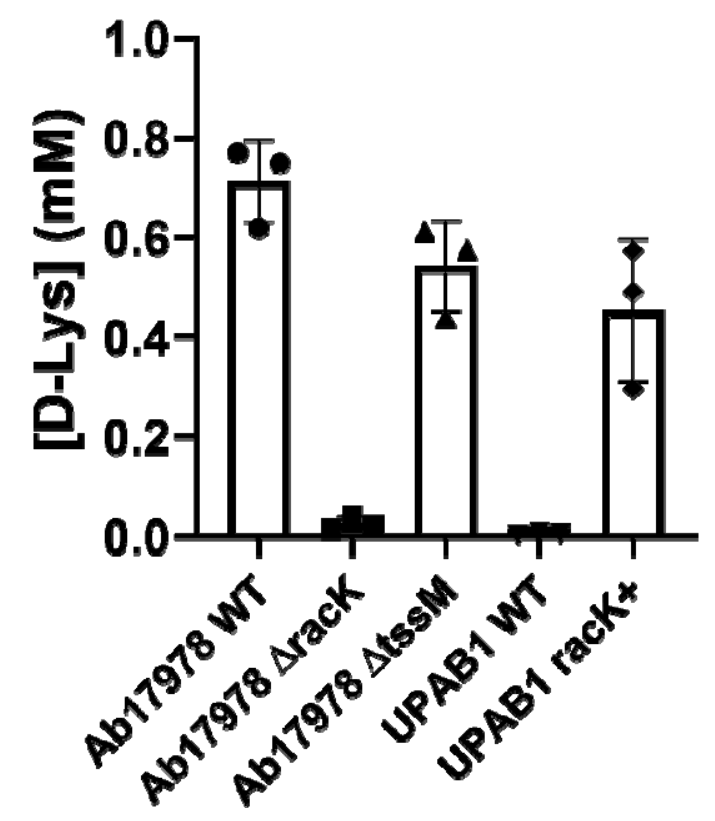

454 Fig. S2: Secretion of D-Lys is independent of T6SS activity. Quantification of D-Lys 455 present in supernatant fractions of the indicated strains using the DAO enzymatic assay. Data 456 represent means \pm SD of three biological replicates. 


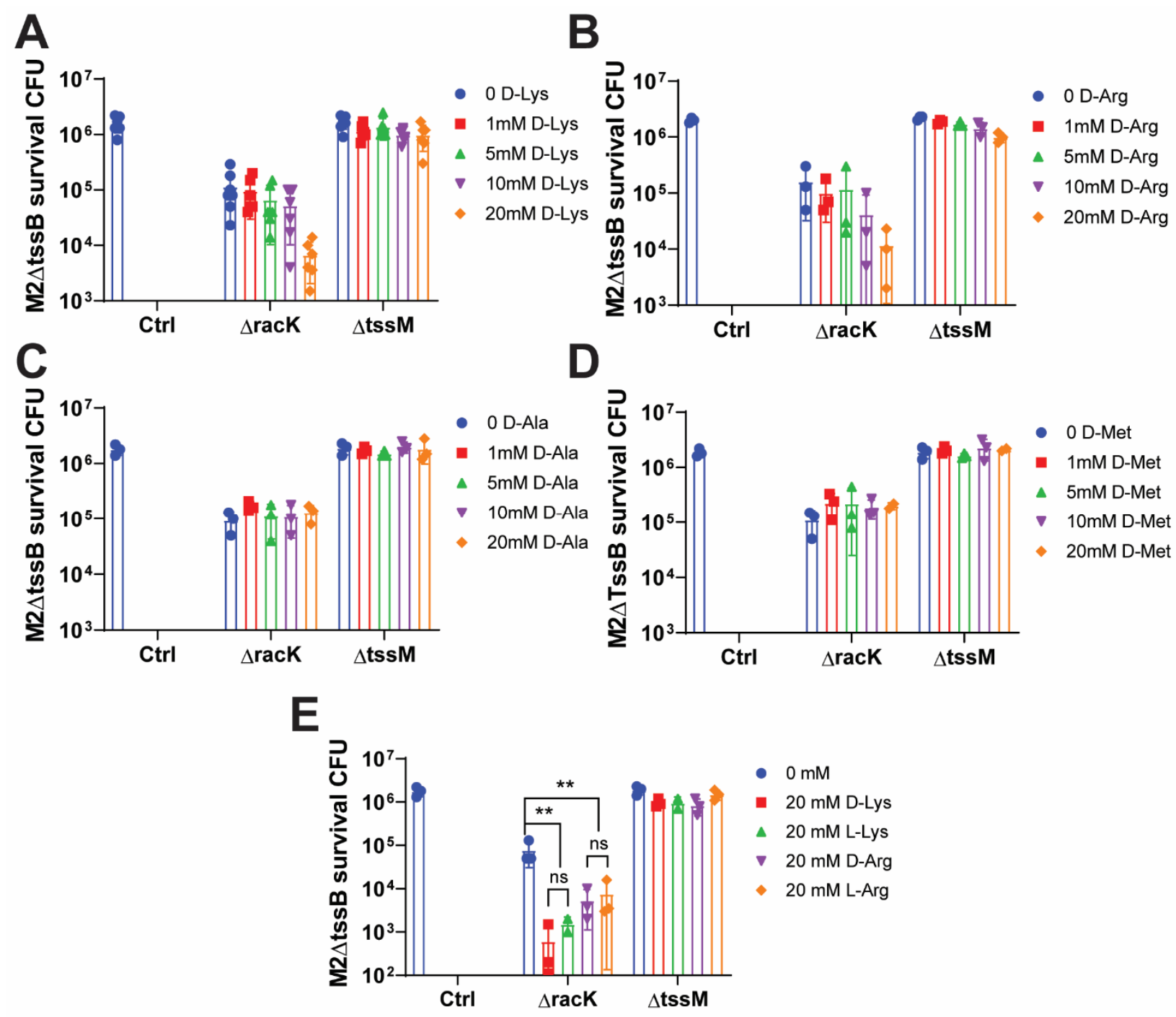

Fig. S3: Extracellular L/D-Lys and -Arg enhance bacterial killing by Ab17978 $\operatorname{racK}$.

458 Competition assay using Ab17978 $\Delta$ racK or $\Delta t s s M$ as predators and M2 $\Delta t s s B$ as prey in LB-

459 agar containing increasing amounts of (A) D-Lys, (B) D-Arg, (C) D-Ala, (D) D-Met, or (E) $20 \mathrm{mM}$

460 of D-Lys, L-Lys, D-Arg or L-Arg. Control (Ctrl) indicates CFU of M2 $\Delta$ tssB growing in LB-agar

461 alone. Bar graphs represent means of prey survival CFU after $4 \mathrm{~h}$ of co-incubation of at least

462 three biological replicates. 


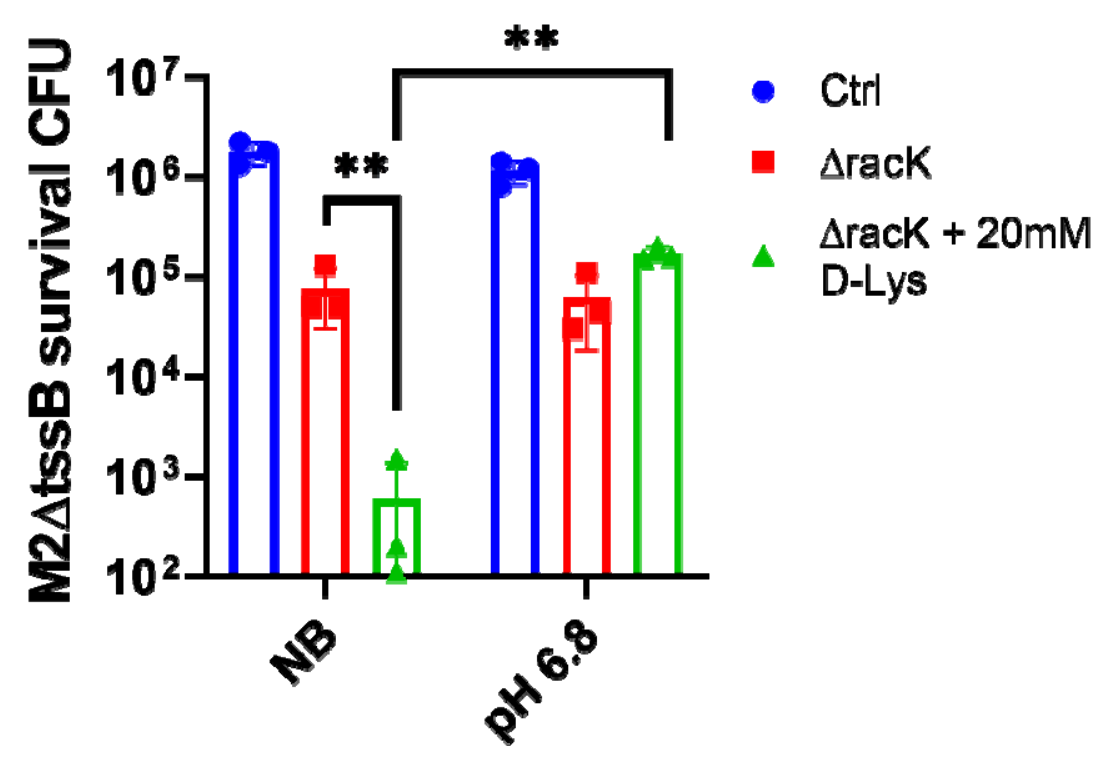

463 Fig. S4: Buffering at neutral pH prevents the synergistic effect of D-Lys on bacterial 464 killing. Competition assay using Ab17978 $\Delta$ racK as predator and M2 $\Delta t s s B$ as prey in LB-agar 465 with or without 20 mM D-Lys in either non-buffered (NB) media or media buffered at pH 6.8. Bar 466 graphs represent means of prey survival CFU after $4 \mathrm{~h}$ of co-incubation of three biological 467 replicates. 


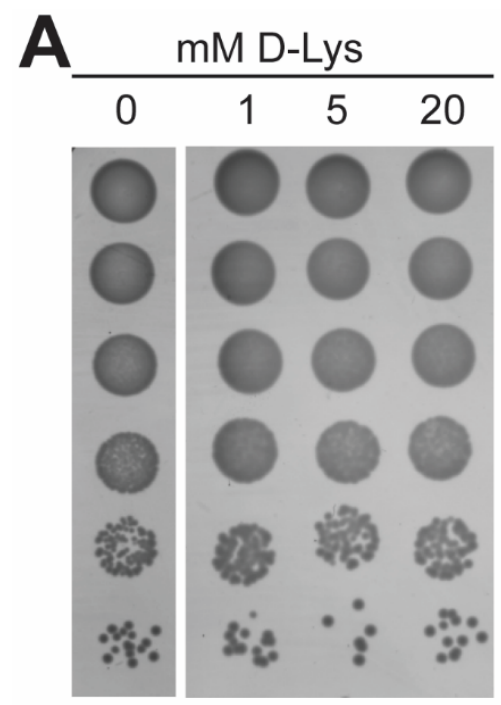

M2 $\Delta$ tssB CFU

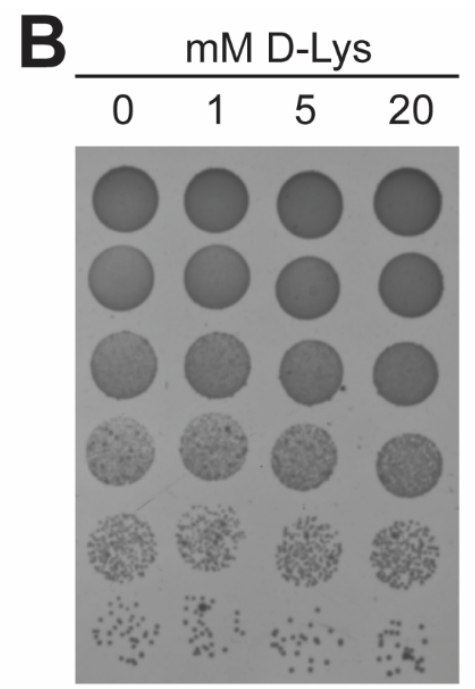

Ab17978 $\Delta$ racK CFU

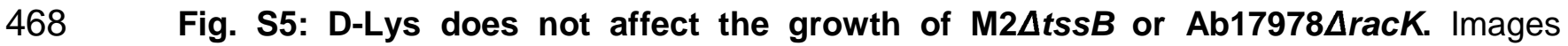

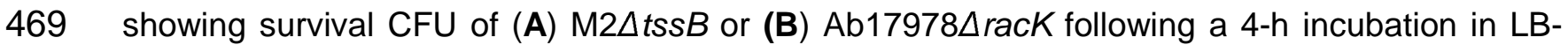

470 agar supplemented with $0,1,5$ or $20 \mathrm{mM}$ of D-Lys on solid LB media. These conditions are

471 identical to those used for competition assays. 


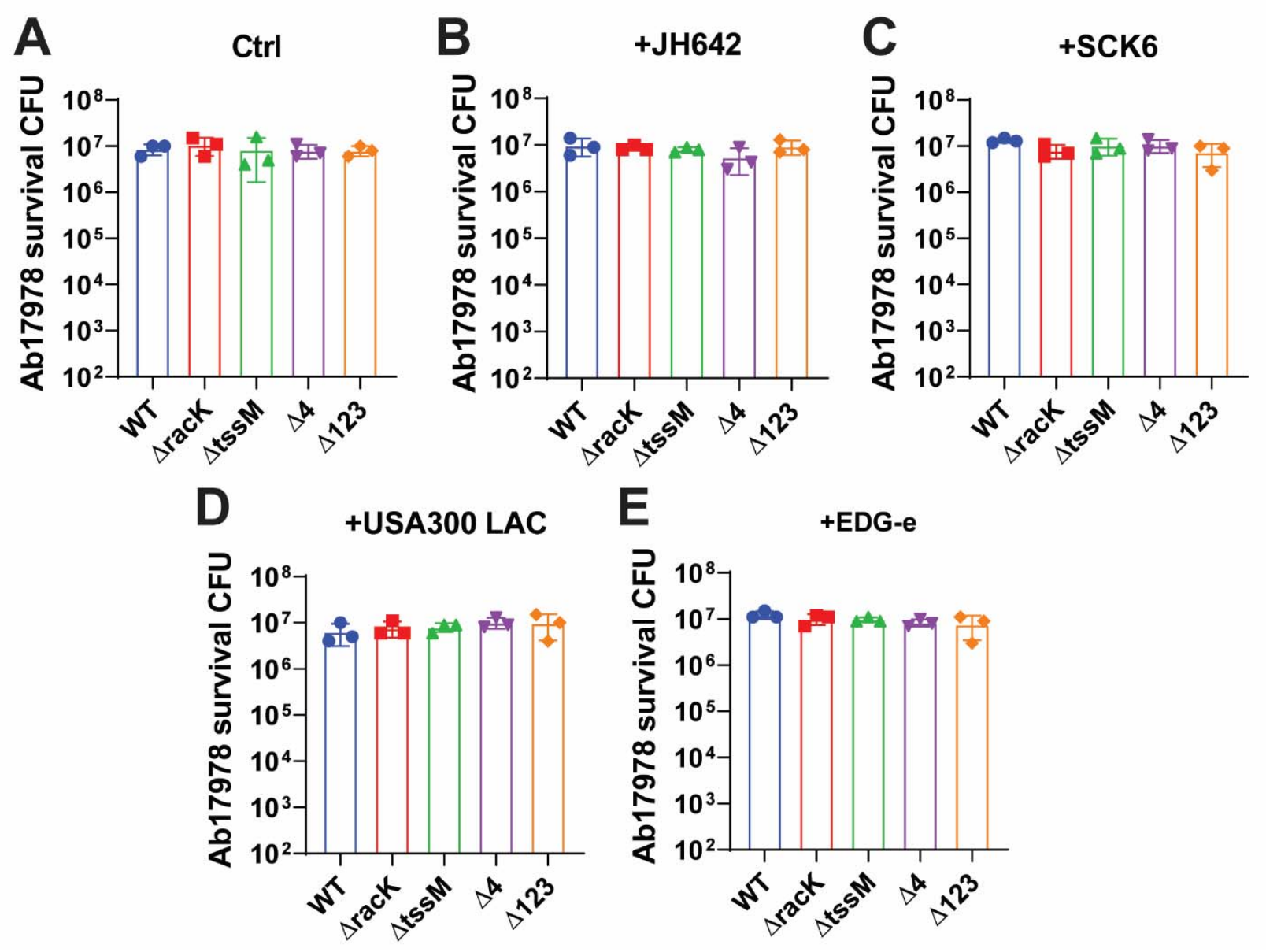

472 Fig. S6: Ab17978 growth is unaffected by co-incubation with various Gram-positive

473 bacteria in solid media. Bar graphs represent means of predator survival CFU after $4 \mathrm{~h}$ of co-

474 incubation with the indicated prey strains (three biological replicates). The samples were 475 obtained during the competition assays shown in Fig. 4. 


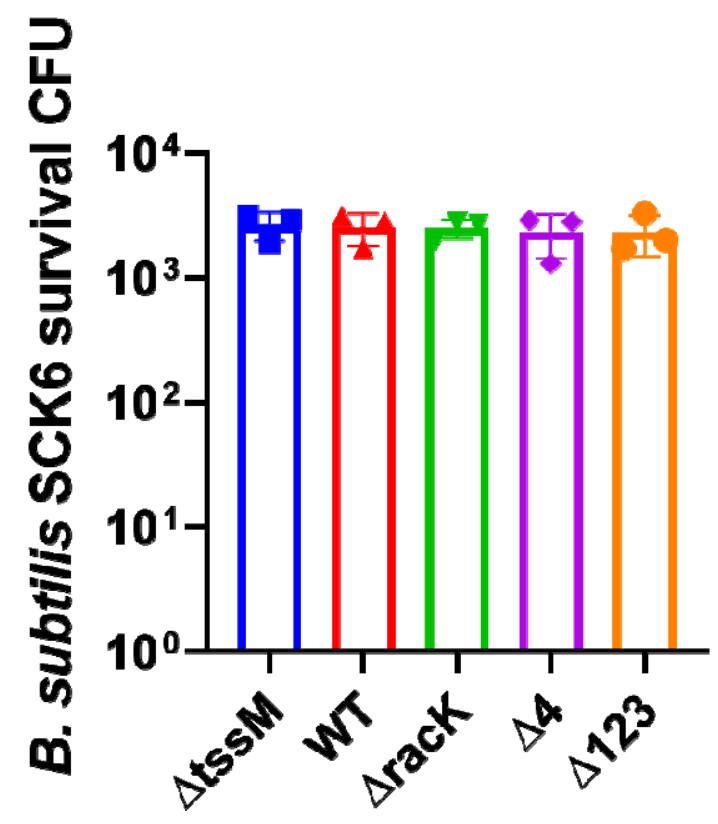

476 Fig. S7: Ab17978 lacks bactericidal activity against B. subtilis SCK6 when co-

477 incubated in liquid media. Interbacterial competition assay in liquid media using Ab17978 WT,

$478 \Delta t s s M, \Delta r a c K, \Delta 4$ or $\Delta 123$ as predators and B. subtilis SCK6 as prey. Bar graphs represent

479 means of prey survival CFU after $4 \mathrm{~h}$ of co-incubation (three biological replicates). 

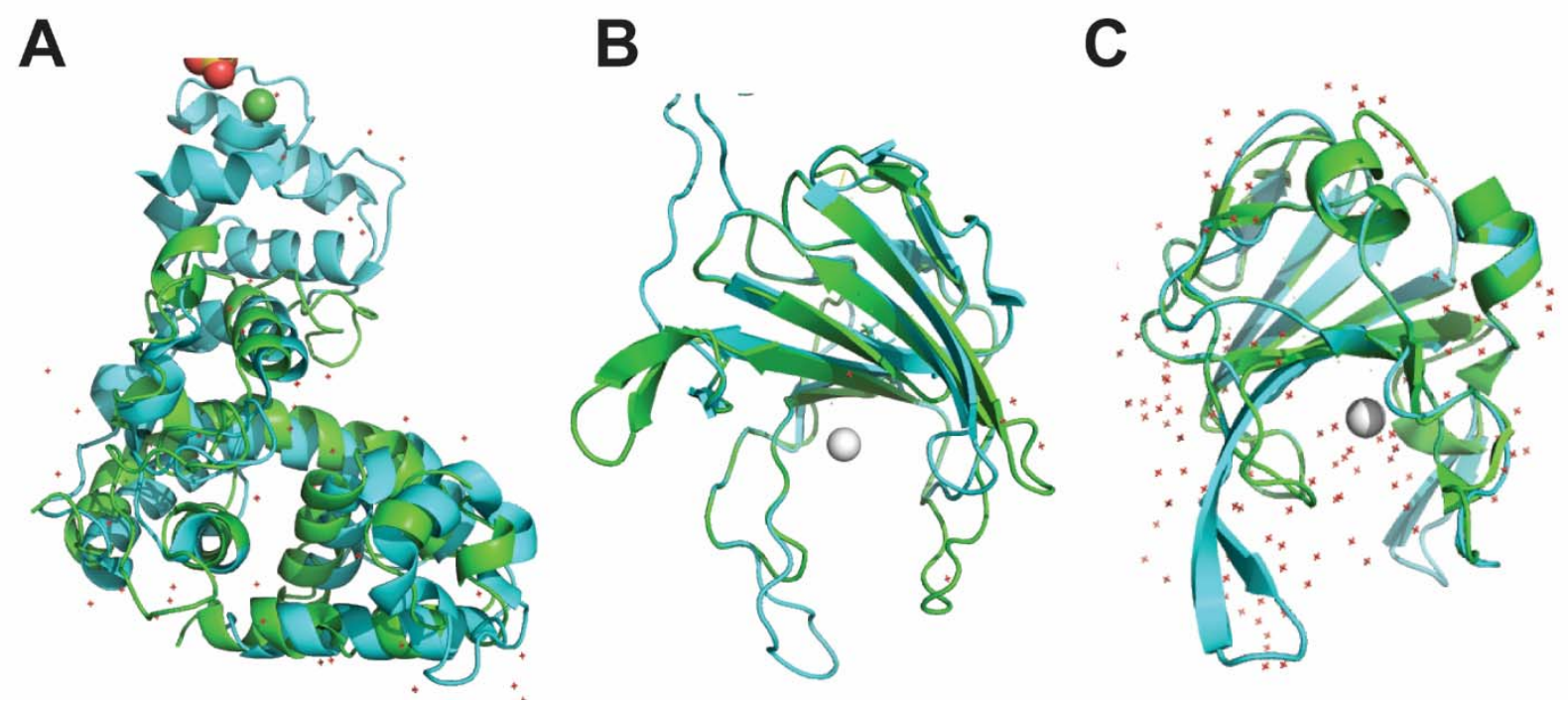

Fig. S8: Tse4 is predicted to be a bifunctional effector with lysozyme and peptidase

481 domains. (A) Tse4 (res. 242-447) modeled to lytic transglycosylase gp144 of bacteriophage

482 phiKZ (PDB: 3BKH). Tse4 (res. 634-798) modeled to (B) the DD-endopeptidase ShyA of $V$.

483 cholerae (PDB:6U2A) and (C) to lysostaphin LytM of S. aureus (PDB: 2B44). Structural models

484 of Tse4 are shown in green; templates are shown in cyan. 


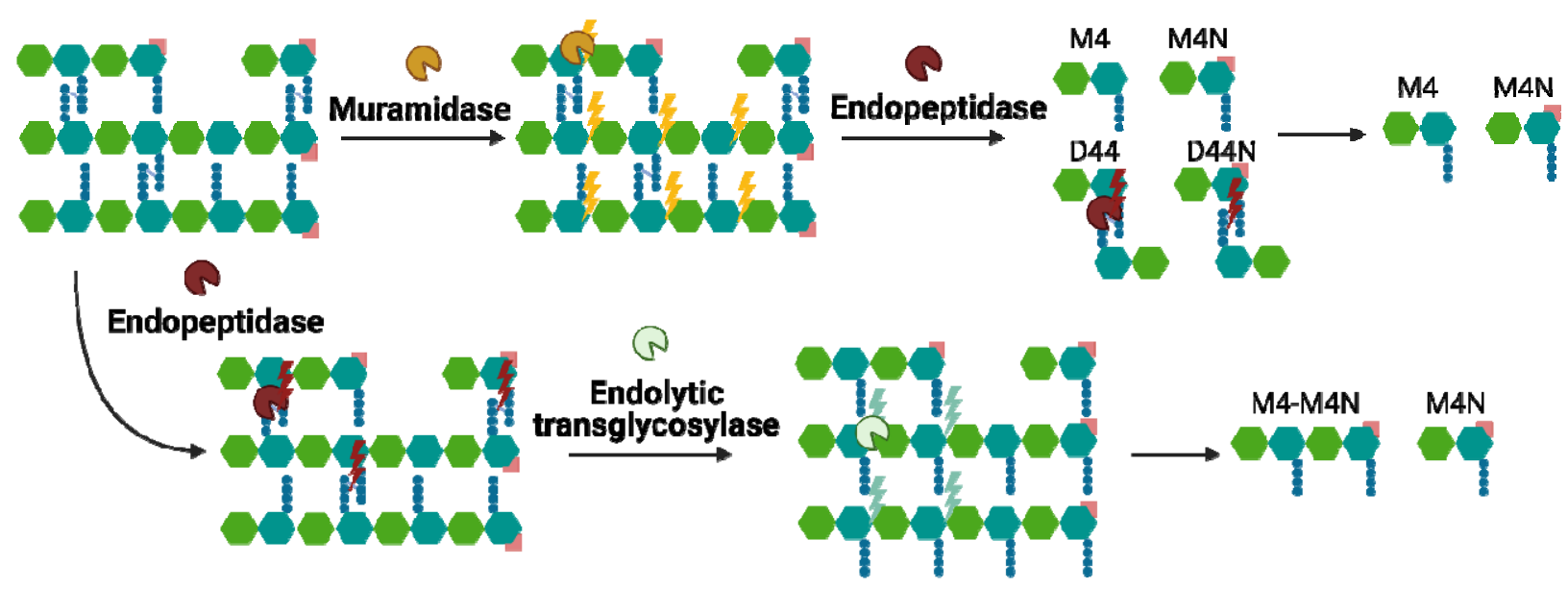

486 Tse4. PG is a heteromeric structure consisting of crosslinked and uncrosslinked muropeptide

487 chains with an anhydromuropeptide marking the end of the glycosidic chain. (top) Muramidase

488 cleaves the glycosidic link between MurNAc (blue) and GlcNAc (green), leading to the release

489 of a mixture of crosslinked (D44) and uncrosslinked (M4) muropeptides, or their anhydro forms

490 (D44N and M4N, respectively). Endopeptidases cleave the peptide stem. Thus, co-treatment of

491 PG with muramidase+endopeptidase is expected to release exclusively uncrosslinked products

492 (M4 and M4N). Like muramidases, endolytic transglycosylases cleave glycosidic bonds.

493 However, unlike muramidases, endolytic transglycosylases form unique anhydromuropeptides.

494 (bottom) Because endopeptidases do not cleave the glycan backbone of PG, endopeptidase

495 digestion of the sacculi generates polymeric MurNAc-GIcNAc chains bearing non-crosslinked

496 stems. In contrast, co-treatment of PG with endopeptidase+endolytic transglycosylase is

497 expected to release large amounts of dimeric and monomeric anhydromuropeptides (M4-M4N

498 and M4N, respectively). 
Table S1: Muropeptide nomenclature and mass for chromatograms shown in Fig. 5.

\begin{tabular}{|c|c|c|}
\hline $\begin{array}{c}\text { (Peak) } \\
\text { Muropeptide }\end{array}$ & $\begin{array}{l}\text { Predicted } \\
\text { mass }\end{array}$ & $\begin{array}{l}\text { Observed } \\
\text { mass }\end{array}$ \\
\hline (1) M4 & 942.4155 & 942.4232 \\
\hline (2) D44 & 1865.8126 & 1865.8698 \\
\hline (3) D44N & 1845.7864 & 1845.8116 \\
\hline (4) M4N & 922.3893 & 922.39284 \\
\hline (5) M4-M4N & 1843.7708 & 1843.7554 \\
\hline
\end{tabular}


Table S2: Bacterial strains and plasmids used in this study.

\begin{tabular}{lll} 
Strain & Plasmid & \multicolumn{2}{l}{ Source } \\
\hline Ab17978 $\Delta$ racK & Ab17978 racK deletion mutant & Le et al. 2020(5) \\
\hline Ab17978 $\Delta$ tssM & Ab17978 tssM deletion mutant & Weber et al. 2016(2) \\
\hline Ab17978 $\Delta \mathbf{4}$ & Ab17978 tse4 deletion mutant & This study \\
\hline Ab17978 $\Delta 123$ & $\begin{array}{l}\text { Ab17978 vgrG1,2,3 gene clusters deletion } \\
\text { mutant }\end{array}$ & Weber et al. 2016 \\
\hline $\begin{array}{l}\text { Ab17978 } \\
\Delta 123 \Delta \text { racK }\end{array}$ & racK deletion on Ab17978 $\Delta 123$ background & This study \\
\hline UPAB1 racK+ & $\begin{array}{l}\text { UPAB1 with chromosomal insertion of racK } \\
\text { and its 300 bp upstream region }\end{array}$ & Le et al. 2020 \\
\hline $\begin{array}{l}\text { UPAB1 } \text { racK++ } \\
\Delta \text { tssM }\end{array}$ & UPAB1 racK+ tssM deletion mutant & This study \\
\hline M2 $\Delta$ tssB & pVRL2 & $\begin{array}{l}\text { Carruthers } \\
\text { 2013(10), this study }\end{array}$ \\
\hline M2 $\Delta$ tssB & pWH1266 al. \\
\hline M2 $\Delta$ tssB::tsi4 & pWH1266-promlac-Tsi4_Ab17978 & This study \\
\hline MG1655 & pVRL2 & This study \\
\hline JH642 & pBAV1k-t5-gfp & This study \\
\hline SCK6 & pBAV1k-t5-gfp & This study \\
\hline EDG-e & pBAV1k-t5-gfp & $\begin{array}{l}\text { Gift from Dr. Ichiro } \\
\text { Matsumura }\end{array}$ \\
\hline USA300 LAC::Erm & & This study \\
\hline
\end{tabular}

\begin{tabular}{l|l} 
Plasmid & Description \\
\hline pWH1266 & ATCC 77092, tetracycline resistance marker \\
\hline pWH1266-promlac- & promotor promlac from pBAV1k-t5-gfp, tsi4 gene from Ab17978 on \\
Tsi4 Ab17978 & pWH1266 backbone, tetracycline resistance marker \\
\hline pBAV1k-t5-gfp & published vector(12), kanamycin resistance marker \\
\hline pVRL2 & published vector(13), gentamicin resistance marker \\
\hline
\end{tabular}


Table S3: Primers used in this study.

\begin{tabular}{|c|c|}
\hline Name & Sequence $3^{\prime}-5^{\prime}$ \\
\hline pWH-Ab17978 Tsi4 Fwd & AGAAAGAGGAGAAATACTAGATGATTAAGTATAAATTATGTC \\
\hline pWH-Ab17978 Tsi4 Rev & CGCCTATTTTTATAGGTTACTATTTTAAAGAATATGAATAC \\
\hline pWH linear Fwd & TAACCTATAAAAATAGGCG \\
\hline pWH linear Rev & AAATGTGCGCGGAACCCC \\
\hline pWH screen Fwd & AAACAAATAGGGGTTCCGCGC \\
\hline pWH screen Rev & AATTCTTGAAGACGAAAGGGC \\
\hline AB RacK Fwd & ATAAAACAAAGTTTCGGATG \\
\hline AB RacK Rev & TCCAGCCTACACAATCGCGAGTTTTTTAATCTTTCCTGG \\
\hline CD RacK Fwd & TAAGGAGGATATTCATATGTAAACTTTAGGTGAATTGATAAG \\
\hline CD RacK Rev & ATGCTGCGCATATTGTTCC \\
\hline AB UPAB1 TssM Fwd & GTACTTATTCTTTCAGATCAGACAG \\
\hline AB UPAB1 TssM Rev & GCAGCTCCAGCCTACACAATCGCTTCTATTCTCGTTCTAAGTTCAAAG \\
\hline CD UPAB1 TssM Fwd & $\begin{array}{l}\text { CGGAATAGGAACTAAGGAGGATATTCATATG- } \\
\text { CATGGAAACTAACCTCTACATTCAAG }\end{array}$ \\
\hline CD UPAB1 TssM Rev & GACCTGAACTGTCTTCGCTC \\
\hline P1 & AGCGATTGTGTAGGCTGGAGCTG \\
\hline P2 & CATATGAATATCCTCCTTAGTTCCTATTCCG \\
\hline AB Tse4 Fwd & AATTTCGTTAGCGCGTTGCAG \\
\hline AB Tse4 Rev & TCCAGCCTACACAATCGCCTTATCCCCCTTATTATTTATCAAACGG \\
\hline CD Tse4 Fwd & ATATATGATTAAGTATAAATTATGTCTGGC \\
\hline CD Tse4 Rev & TGAATTATTATTTGACACAGG \\
\hline
\end{tabular}




\section{Additional references:}

500 1. A. T. Tucker, E. M. Nowicki, J. M. Boll, G. A. Knauf, N. C. Burdis, M. S. Trent, B. W.

501 Davies, Defining Gene-Phenotype Relationships in Acinetobacter baumannii through 502 One-Step Chromosomal Gene Inactivation. MBio. 5, e01313-14 (2014).

503 2. B. S. Weber, S. W. Hennon, M. S. Wright, N. E. Scott, V. de Berardinis, L. J. Foster, J. A. 504 Ayala, M. D. Adams, M. F. Feldman, Genetic dissection of the type VI secretion system in 505 Acinetobacter and identification of a novel peptidoglycan hydrolase, TagX, required for its $506 \quad$ biogenesis. MBio. 7 (2016), doi:10.1128/mBio.01253-16.

507 3. B. S. Weber, P. M. Ly, M. F. Feldman, in Methods in Molecular Biology (Humana Press $508 \quad$ Inc., 2017; https://pubmed.ncbi.nlm.nih.gov/28667630/), vol. 1615, pp. 465-472.

509 4. F. W. Studier, Protein production by auto-induction in high-density shaking cultures 510 (2005), doi:10.1016/j.pep.2005.01.016.

511 5. N. H. Le, K. Peters, A. Espaillat, J. R. Sheldon, J. Gray, G. Di Venanzio, J. Lopez, B. 512 Djahanschiri, E. A. Mueller, S. W. Hennon, P. A. Levin, I. Ebersberger, E. P. Skaar, F. 513 Cava, W. Vollmer, M. F. Feldman, Peptidoglycan editing provides immunity to 514 Acinetobacter baumannii during bacterial warfare. Sci. Adv. 6, 5614-5636 (2020).

515 6. R. Zhou, S. Chen, P. Recsei, A dye release assay for determination of lysostaphin $516 \quad$ activity. Anal. Biochem. 171, 141-144 (1988).

517 7. T. Dörr, F. Cava, H. Lam, B. M. Davis, M. K. Waldor, Substrate specificity of an 518 elongation-specific peptidoglycan endopeptidase and its implications for cell wall 519 architecture and growth of Vibrio cholerae. Mol. Microbiol. 89, 949-962 (2013).

520 8. L. Alvarez, S. B. Hernandez, M. A. De Pedro, F. Cava, in Methods in Molecular Biology 
521 (Humana Press Inc., 2016; https://pubmed.ncbi.nlm.nih.gov/27311661/), vol. 1440, pp.

$522 \quad 11-27$.

523 9. L. Alvarez, S. B. Hernandez, M. A. De Pedro, F. Cava, in Methods in Molecular Biology 524 (Humana Press Inc., 2016), vol. 1440, pp. 11-27.

525 10. M. D. Carruthers, P. A. Nicholson, E. N. Tracy, R. S. Munson, Acinetobacter baumannii $526 \quad$ Utilizes a Type VI Secretion System for Bacterial Competition. PLoS One. 8, e59388 527 (2013).

528 11. A. D. Kennedy, J. B. Wardenburg, D. J. Gardner, D. Long, A. R. Whitney, K. R. 529 Braughton, O. Schneewind, F. R. DeLeo, Targeting of alpha-hemolysin by active or 530 passive immunization decreases severity of USA300 skin infection in a mouse model. $J$. $531 \quad$ Infect. Dis. 202, 1050-1058 (2010).

532 12. A. V. Bryksin, I. Matsumura, Rational Design of a Plasmid Origin That Replicates 533 Efficiently in Both Gram-Positive and Gram-Negative Bacteria. PLoS One. 5, e13244 534 (2010).

535 13. M. Lucidi, F. Runci, G. Rampioni, E. Frangipani, L. Leoni, P. Visca, New shuttle vectors 536 for gene cloning and expression in multidrug-resistant Acinetobacter species. Antimicrob. 537 Agents Chemother. 62 (2018), doi:10.1128/AAC.02480-17. 